University of Louisville

ThinkIR: The University of Louisville's Institutional Repository

Electronic Theses and Dissertations

$12-2014$

\title{
Transfer and the commodification of practice.
}

Kenneth Andrew Smith

University of Louisville

Follow this and additional works at: https://ir.library.louisville.edu/etd

Part of the Creative Writing Commons, Educational Psychology Commons, and the Rhetoric and Composition Commons

\section{Recommended Citation}

Smith, Kenneth Andrew, "Transfer and the commodification of practice." (2014). Electronic Theses and Dissertations. Paper 1738.

https://doi.org/10.18297/etd/1738

This Doctoral Dissertation is brought to you for free and open access by ThinkIR: The University of Louisville's Institutional Repository. It has been accepted for inclusion in Electronic Theses and Dissertations by an authorized administrator of ThinkIR: The University of Louisville's Institutional Repository. This title appears here courtesy of the author, who has retained all other copyrights. For more information, please contact thinkir@louisville.edu. 
TRANSFER AND THE COMMODIFICATION OF PRACTICE

\author{
By
}

Kenneth Andrew Smith

B.A., Minnesota State University Moorhead, 2003

M.A., Middle Tennessee State University, 2007

\begin{abstract}
A Dissertation
Submitted to the Faculty of the

College of Arts and Sciences of the University of Louisville in Partial Fulfillment of the Requirements

for the Degree of
\end{abstract}

Doctor of Philosophy

Department of English

University of Louisville

Louisville, Kentucky

December 2014 

TRANSFER AND THE COMMODIFICATION OF PRACTICE

By

Kenneth Andrew Smith

B.A., Minnesota State University Moorhead, 2003

M.A., Middle Tennessee State University, 2007

A Dissertation Approved on

June 25, 2012

by the following Dissertation Committee:

\begin{tabular}{|c|}
\hline $\begin{array}{l}\text { Dissertation Director } \\
\text { Joanna Wolfe }\end{array}$ \\
\hline Min-Zhan Lu \\
\hline Bruce Horner \\
\hline Susan Ryan \\
\hline
\end{tabular}

Marilyn M. Cooper 


\section{ACKNOWLEDGMENTS}

I would like to thank my dissertation director, Dr. Joanna Wolfe, for her patience with what has admittedly been a rocky process. I can never fully express how much she has helped me over the years. I would also like to thank my other committee members, Dr. Min-Zhan Lu, Dr. Susan Ryan, Dr. Marilyn Cooper, and Dr. Bruce Horner, for their guidance throughout the project. Finally, I would like to express my gratitude for my loving wife, Pamela Elizabeth Mohon-Smith, and my little boy, Kenny Smith, who wants his dad to finish with this project so he can get back to the serious work of playing with toys. 


\title{
ABSTRACT \\ TRANSFER AND THE COMMODIFICATION OF PRACTICE
}

\author{
Kenneth A. Smith
}

December 5, 2014

This dissertation engages in a close reading of the research in composition on transfer, a concept that refers to how the practices learned in one situation influence what a person can do in a future situation. In the studies that have appeared over the last two decades, the results have indicated that not much transfers between writing courses and future contexts, whether they be disciplinary courses or professional workplaces. Yet, despite the increasing prominence of transfer research, little time has been spent discussing the uses and limitations of the concept. To better understand its growing popularity among researchers, I examine its roots in genre theory, its use by empirical researchers, and its function in the debate over first-year composition. Part of the reason we have trouble isolating instances of "transfer" is because of the way the concept frames our understanding of writing development. It treats practices almost like commodities, mental tools that are acquired in the classroom and then eventually carried by learners into new situations.

Following the work of social theorists like Stephen Turner, Jean Lave, and Pierre Bourdieu, I argue for a model of practice that views it as a temporal "coupling" between a person's habitual dispositions and their social environment. The main contribution this 
perspective makes to transfer theory is the emphasis it places on time. Because the world is always in flux, students must continually respond to new challenges, whether they are as simple as adjusting to a difficult professor's expectations or as complex as writing in an unfamiliar genre. If we look at writing development from this perspective, transfer is ubiquitous, for learners must necessarily draw on their previous experiences to meet the demands of a changing world.

The first chapter establishes the dissertation's theoretical framework, focusing especially on Bourdieu's discussion of practice and criticisms made of his work. The second chapter looks critically at the metaphors employed to understand writing practices in genre theory and transfer studies. The metaphors point to some unspoken assumptions in our discipline, mainly by demonstrating the ways researchers portray practices almost like commodities. The third chapter examines the methodological approaches used to examine transfer and shows how researchers often don't fully take into account the tacit dimension of practice. The fourth chapter articulates an alternative model of writing practices, one that brings together the work of practice theory and actor-oriented transfer research. In the final chapter, I suggest some directions for future research, arguing we especially need more information about how students deal with unexpected situations. 


\section{TABLE OF CONTENTS}

PAGE

ACKNOWLEDGMENTS ...........................................................ii

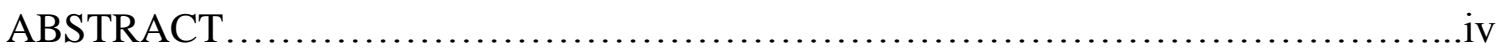

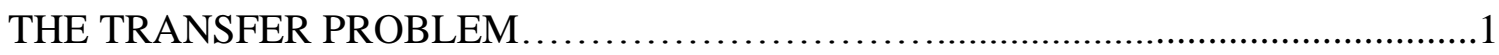

The Importance of Transfer Research......................................6

Social Theory and Transfer Studies........................................11

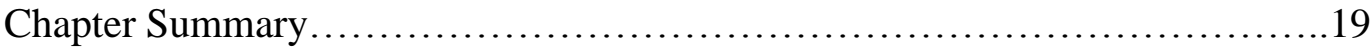

THE COMMODIFICATION OF PRACTICE ....................................23

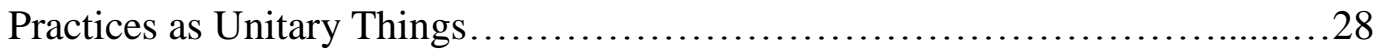

Contexts as Bounded Places...............................................46

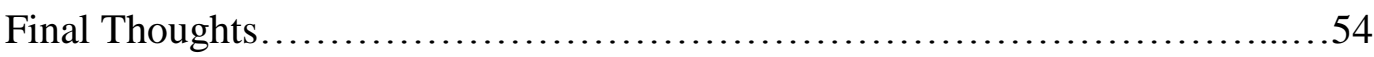

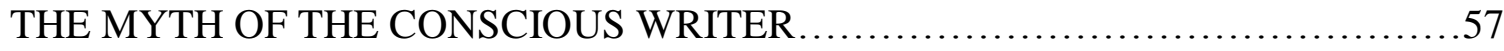

The Duality of the Unconscious-Conscious.................................59

The Trouble with Retrospective Accounts...................................64

The Interpretation of Retrospective Accounts...............................75

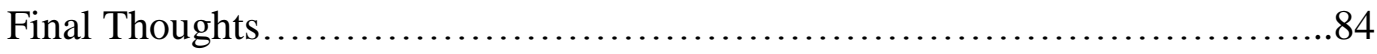

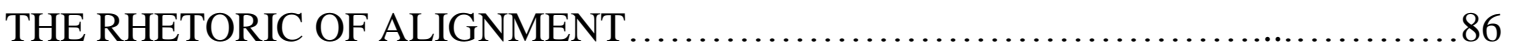

The Doctrine of Sameness............................................... 90

A Model of Practice ............................................................ 96

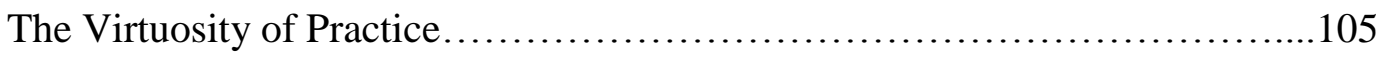


Final Thoughts................................................... 110

DIRECTIONS FOR FUTURE RESEARCH...................................112

Coping with the Unexpected......................................... 114

Conclusion.......................................................... 125

REFERENCES ........................................................... 128

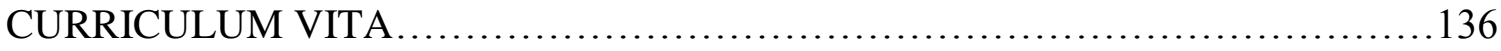




\section{CHAPTER I}

\section{THE TRANSFER PROBLEM}

Over the past decade, a consensus has emerged among many composition scholars that the college writing curriculum is in desperate need of reform. While they are not always in agreement about what needs to be changed, most agree that something needs to be done, particularly in relationship to the first-year composition course. David Smit has argued for the development of a vertical writing curriculum, where advanced composition courses are taught by writing specialists with expertise in a particular discipline. In Reconceiving Writing, Rethinking Writing Instruction, Joseph Petraglia, David Russell, and many others argue for the abolition of the first-year composition sequence. Elizabeth Wardle and Douglas Down have claimed that we should transform the first-year course into an introduction to the composition discipline, partially on the premise that such a course would help students develop some metawareness about how writing varies in different situational contexts. In a similar fashion, Anne Beaufort, Amy Devitt, and Anis Bawarshi want the course to explicitly focus on metacognition, which they claim would encourage the development of rhetorical flexibility. While these proposals vary in their comprehensiveness, most would require a considerable overhaul of the current educational system. To accommodate some of these suggested curricular reforms, administrators would need to change hiring practices, institute new training programs, and rethink disciplinary writing courses. 
As with any call for reform, these proposals are all rooted in a critique of contemporary practices. For the remainder of the dissertation, I will refer to this shared critique as the "transfer problem." According to this family of arguments, the first-year composition sequence was originally developed with the intention of helping students develop a set of general writing practices. Then, when student entered their specific disciplinary courses, they would build upon these foundations. For that reason, many courses focused on grammar instruction, which was viewed as providing students with the invariable rules that governed all writing tasks. The same logic also underpinned the process movement, which argued that the main goal of writing courses should be teaching a process that can be used to undertake any writing task. As Joseph Petraglia explains, this theoretical framework imagined writing "as a masterable body of skills that can be formed and practiced irrespective of the formal context of the writing classroom" (56). After the "social turn" in composition studies, scholars began to raise questions about the existence of such general skills, choosing instead to focus on the unique rhetorical techniques used by different communities. Given how much writing varies between groups, scholars became increasingly skeptical of our ability to teach a single set of skills that would transfer to all situations.

Once we begin to doubt the existence of such "general" writing skills, the mandatory first-year composition sequence becomes difficult to justify. If our goal is to help students help prepare students for the writing they will do in future courses, our task would seem almost impossible — even if we limit our scope to just academic genres, we are still faced with a daunting amount of variation. It has become an open question whether teaching certain "first-year composition" genres helps students write more 
effectively in future courses. To better understand this issue, many composition scholars have turned to transfer studies, a field of educational psychology that Ference Marton characterizes as focused on "how what is learned in one situation affects or influences what the learner is capable of doing in another situation" (499). While educational researchers have utilized numerous methods to examine such questions, many studies involve experiments that present learners with a task, monitor the skills necessary to complete it, and then examine whether they are utilized to solve a similar problem in a different context (Marton 504). These studies have often produced dismal results—in many cases, they determine that little transfer occurs between two situations, which corroborates the idea that our skills are radically context-dependent. Summarizing the history of transfer research, Douglas Detterman claims that studies exhibiting "true, spontaneous transfer are rare if they exist at all" (15). As he explains, the most effective strategy is probably to "teach people exactly what you want them to learn in a situation as close as possible to the one in which the learning will be applied" (17).

While transfer research in composition studies remains in its infancy, our findings have supported a similar conclusion. For the most part, our understanding is limited to longitudinal case studies and other qualitative approaches, but they all indicate the complex, turbulent nature of learning to write. Students don't just master a skill and then apply it in future situations; rather, they embark on what Lee Ann Carroll describes as the "literacy rollercoaster," a process that involves periods of both success and failure (49). The existing studies feature numerous examples of students struggling to understand new tasks, and, in many cases, unsuccessfully drawing upon strategies developed in previous courses (Beaufort; McCarthy; Walvoord and McCarthy). Indeed, the research contains 
few examples of successful transfer of writing skills, and these studies mostly involve relatively small samples of students (Nowacek; Artemeva; Smart and Brown).

Composition scholars have proposed several different ways of addressing the "transfer problem." These curricular reforms can be divided into two broad categories. Scholars in the first group argue for the abolition of the first-year composition sequence in favor of disciplinary writing courses. They buttress this position with a number of other claims. First, they argue that the continued existence of the first-year course promotes certain mythologies about the nature of writing practices; so long as it exists, faculty can continue to believe that writing is a transparent container for ideas, that it just involves learning grammatical rules, and other such problematic assumptions. According to Russell, this myth has benefited the English department by allowing it to serve as the arbitrator of what constitutes "good style"; however, it also "makes it easier for a discipline to assume that when students from previously excluded language backgrounds fail to successfully write in its genres, it has nothing to do with its failure" (67). Once the course has been abolished, other disciplines will have to take more responsibility for the development of writing skills. Secondly, these scholars have argued the first-year course encourages unjust labor practices. As Sharon Crowley points out, first-year composition teachers "usually are paid less, have no access to benefits or job security, have little or no advance warning about what and when they will teach, and have inadequate office and communications facilities" (241). If the course was eliminated, administrators would have no need for the adjunct faculty that fill these positions. Finally, such reforms would liberate our discipline from its association with the course-while this might result in less funding, it would also "free up" scholars to pursue other interests. 
In contrast, scholars in the second group argue the first-year course might still serve a useful purpose. However, it should be given a more humble mission-instead of attempting to teach students a set of universal skills, instructors should focus on developing mindfulness, critical consciousness, or metacognition, a process by which an individual makes a practice's tacit principles more explicit so he/she can make a more conscious choice between the possible options. Support for such a curriculum has come from scholars working in a variety of fields (Beaufort; Canagarajah; Bawarshi; Takyoshi and Selfe). Proponents of metacognition generally argue that it encourages two different dispositions. First, it makes students "rhetorically flexible," more capable of creatively meshing discourses, languages, genres, and modalities to meet the demands of new situations. While they might not immediately know how to respond to future writing situations, students should be able to more quickly develop the necessary skills. Secondly, such metacognitive ability makes students more consciously aware of the political dimension of language. That way, students become aware of how certain practices advance the ideological interests of dominant social institutions and therefore will be more capable of resistance. Scholars in this group have recommended several reforms, including promoting genre awareness, analyzing the uses of different modalities, and creatively meshing different languages. The idea is that we should no longer focus on helping students become more competent in a specific genre but should teach them how to successfully adapt their skills to new contexts.

While these reforms differ in many respects, they both stem from the belief that transfer is an intractable problem. As Smit puts it, "the evidence suggests that learners do not necessarily transfer the kinds of knowledge and skills they have learned previously to 
new tasks. If such transfer occurs at all, it is largely unpredictable and depends on the learners' background and experience, factors over which teachers have little control" (117). Or, in the words of Russell, "writing does not exist apart from its uses, for it is a tool for accomplishing object(ive)s beyond itself . . f from this theoretical perspective, the object(ive) of [first-year writing courses] is extremely ambiguous because those involved in it are teaching and learning the use of a tool (writing) for no particular activity system" (“Activity Theory" 57). To provide one final example, Beaufort argues that first-students are often "ill-prepared to examine, question, or understand the literacy standards of discourse communities they are encountering in other disciplines . . this can result in negative transfer of learning: what worked for a freshman writing essay is inappropriately applied to writing in history, or social sciences, or sciences or in business" (11). Because first-year composition is not attached to a specific discipline, many researchers are enormously skeptical that students can develop skills that will be useful in their future disciplinary courses. My dissertation takes this shared belief in the "transfer problem" as its point of departure. Rather than seeing transfer as a practical problem faced by curriculum designers, I see it primarily as a theoretical problem that has arisen because of how transfer causes us to view the learning process.

\section{The Importance of Transfer Research}

The most beneficial aspect of the contemporary transfer research is how it has brought attention to how individual learners change as they move through the curriculum. Partially because of the "social turn" in composition pedagogy over the last two decades, a considerable amount of attention has been devoted to what genres of writing are done in different institutional settings. One of the reasons that transfer has come to be viewed as 
such a thorny problem is our increasing awareness of the tremendous variety of practices that are classified as "writing" in the disciplines. In a series of studies done by Amy Devitt on tax accountants, she reveals the ways that novice writers develop reports on legal matters by fully reproducing large chunks of text from relevant tax codes. Jeanne Fahnstock and Marie Secor have shown the way researchers employ special topoi, which are specialized forms of argumentation unique to certain contexts. In their work on literary studies, they have shown how scholars unconsciously draw on numerous strategies, such as the idea that a deeper, more complex reality exists beneath the surface of texts. In one particularly interesting study, Peter Medway examined what he describes as the "fuzzy genre" of architecture students' sketchbooks, which feature everything from detailed illustrations to inspirational poems. The actual texts varied considerably in almost all respects, including everything from their physical format to their actual content. Some of the books were little more than sequences of drawings, while others included almost no sketches and relied mostly on written text. They sometimes contained business cards nestled in the pages and other documents collected over the years. As Medway observes, part of the appeal of genre theory is the idea that genres act as institutional "shortcuts" that individuals can deploy to navigate reoccurring social situations. When faced with texts like architectural notebooks, the question arises of how the genre can possibly serve as a "mental shortcut" due to the amount of variability in the finished products.

"Fuzzy genres" are instances where transfer theory might clearly illuminate aspects of writing that are missed by approaches that focus primarily on how texts are used by social groups. As Medway observes, what is perhaps most interesting about the 
architectural notebooks is how students actually went about learning to produce them. One set of students seemed to use "procedures [that] had been carried over into this context from their experience of taking notes in notes in conventional academic lectures" (134). Another student borrowed from "the DayTimer organizer that he had previously kept and that had proved too small for the expanded range of inscriptional demands that now had to meet" (134). One student even borrowed from practices learned from her French high school education. In many ways, these notebooks vividly demonstrate how students deploy skills that they learned in previous contexts creatively to tackle problems in new settings. Transfer research brings with it a particular focus on the individual student and how they change as they move throughout their lives. Rather than seeing the texts they produce as solely the products of being socialized into a particular community, transfer encourages us to see learning as the process of cobbling together what we've learned to overcome new challenges. Each new social setting becomes simply one small part of the story of how one learns to write - a process that began when the student spoke his/her first word, that continued through elementary and high school, that happens during conversations with friends, that occurs while reading political blogs and watching television, that will proceed into future university courses and professional careers, and that ultimately will have a scope that extends far beyond our classroom.

My dissertation sprung from the desire to retain transfer research's focus on the development of the individual learner while clearing the theoretical debris that makes the writing classroom seem like such a fraught institution. Following in the tradition of neopragmatist theorists such as Richard Rorty, my interest here is more in "dissolving inherited problems rather than in solving them" (20). As he perceives it, the goal of the 
abstract theorizing that I see myself undertaking in this dissertation is not the discovery of a secret hidden reality missed by researchers; rather, it's an attempt to argue that we should consider discarding our old tools and using new ones. As Rorty puts it, "to come up with such a vocabulary is more like discarding the lever and the chock because one has envisaged the pulley, or like discarding gesso and tempera because one has now figured out how to size a canvas properly" (12). My claim is that the perennial failure of researchers to detect "transfer" between disciplines is not the product of an intrinsic limitation of the human mind but rather because of the vocabulary being employed by researchers. I want to suggest that we won't resolve the difficulties uncovered in recent transfer studies by simply looking harder or gathering more data or performing more longitudinal research. My suspicion is that what we are currently imagining as "transfer" is happening all the time right underneath our noses, and how to see it is to look for it in a different way.

I will confess that I find myself reaching for a new vocabulary partially for political reasons. I'm not the first to be troubled by the conclusions that transfer research seems to draw about the students that populate our classrooms. As Daniel L. Schwartz, John D. Bransford, and David Sears argue in "Efficiency and Innovation in Transfer," the classic definitions and measures of transfer have the troubling effect of making learners “look dumb." Initial transfer studies placed learners in sequestered problem solving environments that were designed to eliminate the "competing influences" of the skills that they were provided in previous settings (5). Research subjects usually were tested on their ability to solve abstract logic puzzles that had little relationship to the skills and competencies that they have developed over a lifetime of education. Often, transfer 
studies ignored the sophistication of the practices actually employed by individuals in their effort to trace the impact of a particular educational experience. Jean Lave demonstrates this point quite vividly in Cognition in Practice, where she examines the mathematical procedures used by supermarket shoppers to decide on the best purchase. As she points out, little correlation existed between their ability to successfully navigate the supermarket and their aptitude at solving traditional mathematical problems. She concludes that "the success and frequency of calculation in supermarket and simulation experiments bear no statistical relationship with schooling, years since schooling was completed, or age" (57). While it might be tempting to conclude that their formal mathematics training had little effect on their everyday experiences, they might be drawing on their schooling in subtle ways that are more difficult to detect. Schwartz, Bransford, and Sears have suggested that school-taught mathematics might enable people to "invent or more quickly learn new techniques that were suited to a shopping context" (5). When we imagine learning as little more than the direct application of a skill in another context, we risk missing the ways learners innovate their practices to cope with new challenges.

While composition scholars rarely takes the "experimental" approach popular in educational research, the fact that we've arrived at some of the same conclusions should at least give us pause. Linda Bergmann and Janet Zepernick paint a picture of student learners that are ruthlessly seeking to improve their grades and hold obviously problematic views about the English discipline. Lucille Parkinson McCarthy describes the student in her study as a "stranger in strange lands" who "believed that the writing he was doing was totally unlike anything he had done before" (126). Anne Beaufort 
characterizes one student as using arguments that were "poorly supported" and unable to recognize that history professors want "focused, linear arguments" (104). My concern is that these studies fail to recognize the insightfulness and creativity that seems evident to those of us who work daily as instructors of college-level writing students. Perhaps more importantly, they seem to neglect the abilities that seem evident in other studies, such as the rich array of resources that Medway's architectural students use in their sketchbooks. Because my argument hinges on the fact that looking at the data in a different way might cause readers to notice other aspects of the learning process, a considerable amount of the dissertation will be spent revisiting these transfer studies and rethinking some of their main conclusions.

\section{Social Theory and Transfer Studies}

I see my work fitting into a tradition of social theory that can ultimately be traced back to Pierre Bourdieu. While he devoted his attention to a variety of subjects, he consistently maintained that his work was an attempt to move beyond the traditional divisions that plagued social science, particularly the distinction between what he calls the "subjective" and "objective" methods of sociological investigation. The "subjective" theorists - by which he was mainly referring to the early phenomenologists—strove to describe the social world as it was actually lived by their participants. While he sympathized with this project, he felt that such research tended to emphasize the "taken for granted" aspects of experience; such theorists failed to connect their subjects' daily lives to larger social structures, such as economies, systems of domination, and language. On that score, "objective" researchers were more on target. Their approach overcame some of subjectivism's problematic tendencies, but its success largely came from 
ignoring the lived experience of its participants. In particular, it failed to acknowledge the role scientific investigation itself played in research; after all, sociological models are not literal descriptions of the lives of individuals but rather means for translating practices into scientific discourse. People don't actually behave in such a logical fashion-as he puts it, "One can say that gymnastics is geometry so long as this is not taken to mean that the gymnast is a geometer" (Logic 93). Objective theorists ignore that individuals must continually adjust to the world's shifting demands. Sociologists must connect individual behaviors to social structures, but they also must never forget the chaotic nature of our lives. To avoid confusing our models with reality, we must therefore consider the interaction between the subjective and objective elements of experience.

Drawing from the metaphor of team sports, Bourdieu invites scholars to imagine social structures like a field. Just as a game's players are positioned in relation to their competitors, we are also embedded inside a network of shifting relationships (Logic 6667). We are locked in a struggle for the most advantageous position, much like the football player who switches directions to fool his opponents. The notion of a field captures the sense that social arrangements, such as composition classrooms, are not uniform entities but rather plurlalized structures composed of multiple individuals. When we discuss the university, we have a desire to organize it neatly into disciplines, even if this classification system is just a convenient fiction. After all, a discipline is composed of numerous individuals, all of which are related to each other in a series of fluctuating power relationships. From a distance, their practices might seem to cohere, but a closer examination reveals that each individual is always jockeying for position; he/she is responding to others through journals and electronic bulletin boards, seeking for a way to 
leverage his/her expertise to his/her advantage within the immediate social network. In that sense, saying students are "acquiring a disciplinary discourse" ignores the practical reality, which is that students are responding to particular individuals-namely, the professors and students in their courses. Naturally, each of these individuals will have their own peculiar tendencies, which means that students are forced to continually adjust their practices to meet these changing demands.

An individual's behaviors are locked in a dialectical relationship with the field. Other social actors partially determine the shape of the individual's actions, for he/she is continually responding to their feedback. At the same time, the individual shapes the field, for individual group members determine the boundaries of a social space. For example, people are expected to wear certain clothes to formal events, although what is viewed as appropriate varies among different individuals. Bourdieu's term for these cognitive dispositions is the habitus, which he defines as systems of durable, transposable dispositions, structured structures predisposed to function as structuring structures, that is, as principles which generate and organize practices and representations that can be objectively adapted to their outcomes without presupposing a conscious aiming at ends or an express mastery of the operations necessary in order to attain them. (Logic 53)

These systems are structured in the sense that they are developed through our interaction with the field. As we respond to the actions of other group members, we gain an intuitive sense of how to move about the social space. The habitus is structuring in the sense that it organizes how individuals perceive the world. To successfully play a game, a person 
must know how to respond to other players. To use one of Bourdieu's favorite phrases, an individual must develop a "feel for the game." Much like the shape of the field is determined by the habitus of the group, an individual's habitus is shaped by how he/she adjusts his/her dispositions to the behaviors of the field.

Due to the confusion of Bourdieu's ideas with other structuration theorists, the notion of habitus has been criticized as deterministic, even though this interpretation is at odds with his own belief that the idea of habitus is what allows him to escape such difficulties (Giroux; Nash; Jenkins; Turner, "Mirror Neurons"). The perplexing nature of the habitus has caused some scholars to argue that Bourdieu advances two incompatible social theories. In "Thinking with Bourdieu against Bourdieu: a 'Practical' Critique of the Habitus," Anthony King divides Bourdieu's ideas into what he describes as his "practical theory" and the habitus. As he argues, the "practical theory" emphasizes that "social agents are 'virtuosos' who are not dominated by some abstract social principles but who know the script so well that they can elaborate and improvise upon themes which it provides and in the light of their relations with others" (419). In contrast, the habitus specifies a unified collective entity — when people are socialized into a group, they acquire a set of perceptual dispositions shared by all its members. In King's view, the habitus is therefore no different than the other collective entities that have posited throughout the history of social theory. People are sometimes viewed as sharing a set of "presuppositions" or "tacit rules" that guide their actions. In composition studies, researchers commonly view people as operating under a shared "discourse" that shapes their perspective of the world. The problem with these collective entities is that they 
inevitably open up the charge of determinism, largely because it is difficult to see how people might change their practices if they are produced by a shared social entity.

When these collective entities are examined in more detail, their operation ultimately seems mysterious. As Stephen Turner argues in The Social Theory of Practices: Tradition, Tacit Knowledge, and Presuppositions, the problem emerge the moment we try to imagine how "discourses" or "tacit rules" are transmitted between people - after all, previous generations don't just open up our brains and "program" us with a set of social practices. In Brains/Practices/Relativism: Social Theory after Cognitive Science, Turner claims that such theories adhere to the "snap-on" model of the learning process. The idea is that "the student tries this and tries that ... and does all of this without understanding. But at some point something clicks, and the student 'has' the rule" (Brains 29). Because learning always takes place either through the medium of language or from direct observation, it seems impossible for practices to ever be perfectly transmitted through such a process. For that reason, social theorists have historically resorted to positing the existence of transcendental entities - such as social structures or the collective conscious - that are tapped into by learners. The idea is basically that practices are downloaded from a mystical database. Of course, people are not literally connected by cables to a computer network, so the question arises of where we might locate these transcendental objects. While Bourdieu also questions the existence of collective entities in his criticism of the "objective" structuration theorists, he also sometimes lapses into language extremely reminiscent of these views. He talks about how practices are "reproduced" almost like people are run through a transcendental copy machine. At other times, he vaguely talks about perceptual "schemes" that somehow 
guide our actions. As Turner points out, even his dominant metaphor-the idea that practices are something like a game-seems to rely on the existence of collective entities, for games are after all structured by rules (“Mirror Neurons” 351).

While certain passages open him up to this criticism, Bourdieu emphasizes at several points that he never intended the habitus to be viewed as a unified social structure. In fact, when Bourdieu addresses the relationship between individual and collective habitus, he directly states that a collective habitus should not be viewed as a shared entity. As he explains, one mistaken way to understand the habitus is to imagine it as a "subjective but non-individual system of internalized structures" (Logic 60). He ultimately rejects this position because it would make practices "impersonal and interchangeable" (60). Instead, he argues an individual habitus shares a set of family resemblances with the larger habitus — as he puts it, "the singular habitus of members of the same class are united in a relationship of homology, that is, of diversity within homogeneity characteristic of their social conditions of production" (60). He emphasizes that the reason people exhibit similar behaviors is not because they "acquire" something that shapes their perceptions. Two people are not similar because they share a languageon the contrary, their linguistic practices are the product of cognitive dispositions developed in similar social conditions.

To help bring some clarity to this admittedly abstract argument, it might help to think through what we mean when we say that people "share" a set of practices. One way of understanding this sentence is to say that we share something in common inside our minds like a discourse. Another way of understanding it is to say that we engage in what seems like similar behaviors even though what is producing those actions in our brains 
might actually be extremely different. Consider the process of learning how to dance. When we all arrive at the dance studio the first time, we all come from different places, with some of us perhaps having a considerable amount of experience while other might have never even done anything resembling a dance move. An instructor might begin by establishing a set of explicit guidelines: he/she might discuss positions of the feet, general principles for moving the hips, and offer other such "tips." Of course, we all will react to these instructions in different ways, with some of us picking up on them quickly while others lapse into confusion. Then, the instructor might pair us up with other people, and some of us will learn more effectively by working with our partners while others might feel anxiety about the social interaction. After many years of dancing education, though, we might all arrive at what most people would qualify as "competent dancing skills." However, the careful observer will notice subtle signs that what is going on inside our heads is actually substantially different, perhaps by observing the unique styling that we add to a particular technique.

Looking at practices in this way means acknowledging the importance of time. Because the social world is always in flux, we must also acknowledge the role difference plays in our lives. As we move throughout the day, we are continually confronted with an array of new challenges, which leave what Turner describes as subtle "mental traces" that influence the future direction of practices. We might have to ship off an e-mail to customers at a new business, many of which we only briefly met at a company event. We might be faced with a familiar task but find ourselves in a different mental state, perhaps due to our emotions after receiving some bad news. Due to our continual encounters with different situations, innovation must paradoxically be routine. Furthermore, as Bourdieu 
argues, innovation doesn't necessarily require conscious intervention, whether it is metacognition or one of its variants. Innovation is built into practice-without the ability to react to changing circumstances, we would be nothing more than social robots. As he explains, "To reintroduce uncertainty is to reintroduce time, with its rhythm, its orientation and its irreversibility, substituting the dialectic of strategies for the mechanics of the model, but without falling over into the imaginary anthropology of 'rational actor' theories" (Logic 99). Many binaries that plague our discussion of the mind break down in Bourdieu's system. Rather than make a sharp distinction between automatic routines and conscious innovation, he imagines both as fundamental to practice and therefore triggered at all levels of awareness.

Naturally, not all practices are perceived as having the same value. In the case of language, different ways of speaking are viewed as being more respectable in certain situations. To describe these unequal power relationships, Bourdieu uses the term capital. Just as material goods are assigned different prices, practices also have a particular cultural worth. In some cases, they can be directly exchanged for economic capital, such as when a college graduate uses certain skills to obtain a job. To use one of Bourdieu's examples, he argues in Language and Symbolic Power that the bourgeois express disdain for language associated with the body, such as "coarse" jokes and "crude" comments (87). For the upper class, using such language is a sign of poor taste. On the other hand, when politicians want to negotiate with the "regular folk," they might use such language to seem more "in touch" with the public's concerns (68-69). Part of mastering a game involves understanding the shifting values assigned to different strategies in the marketplace. 
One of the main advantages of Bourdieu's framework is how it sets our social lives in motion. Certainly, some fields privilege routine, but they are the exception. While Bourdieu acknowledges the importance of habits, he views them as only a component of practice. As he explains in Distinction: A Social Critique of the Judgment of Taste, practice might be best defined through the formula, "[(habitus)(capital) $]+$ field $=$ practice." (101). By this, he means that the term refers to how dispositions are deployed in response to particular social arrangements. A practice is precisely this dialectical relationship between cognitive dispositions and social structures. Bourdieu imagines individuals as more than helpless creatures programmed by the surrounding culture. Rather, they are virtuosos whose cognitive dispositions always allow for "regulated improvisation" (Outline 79). In this shifting social landscape, a successful social actor must do more than rigidly employ the same routines, lest they be outplayed by other group members. He views individuals as continually adjusting their practices in response to other individuals.

\section{Chapter Summary}

The second chapter argues the transfer concept causes researchers to portray practices as commodities. While scholars rarely explicitly defend this position, it can be seen primarily through their use of metaphors - in many cases, practices are represented as physical things that learners "transfer" between physical locations, almost like a truck shipping a product to a department store. The problem with this perspective is that it fails to capture several characteristics of social practices. In particular, it neglects the way that they often overlap and share resources — while it might seem that writing a business memo and a short story are distinct activities, they both at least draw on certain basic 
syntactic skills, such as composing paragraphs and coherent sentences. Also, this model provides a limited sense of how learners change over time. For example, if practices are imagined as tools, then the only way for a student to learn is to acquire a new tool. As a result, we frequently ignore how practices are transformed as students encounter new situations. Due to many of these theoretical preconceptions, researchers have come to see transfer as an intractable problem, mainly because they are unable to see how the "tools" acquired in a particular situation can be directly applied to the writing tasks in a different context.

The third chapter argues that current research has not adequately taken into consideration the tacit dimension of writing practices, partially due to the lack of a model for understanding the relationship between unconscious and conscious cognitive processes. Drawing from the work of social theorists, phenomenologists, and expertise researchers, I forward a model of the mind that sees the unconscious and conscious as embedded in a dialectical relationship. As I point out, even if students claim that they learned nothing valuable from their writing courses, they might have developed several practices without their conscious awareness. Therefore, if we limit our research to just methodologies that ask for retrospective accounts from our students, we risk only developing a limited sense of what they learned from their courses. As an alternative, I propose that we use a wider range of methodologies, including those that directly look at composing behaviors, such as collecting student papers, examining revisions, and conducting classroom observations. However, we not only need to broaden the types of data that we collect, we also need to change how we interpret what our students say about their experiences in writing courses. When students express skepticism about what they 
learned in their FYC course, we must recognize how various cultural ideologies influence their opinions. In many cases, they might reflect their participation in peer groups and disciplinary communities that view the English profession as involving primarily "fun" writing activities with no relevance to the workplace. We need to be especially careful of how we interpret accounts of student struggles, for they may simply reflect the discomfort that all learners feel when they must adjust to a new practice.

The fourth chapter attempts to overcome some of the shortcoming of traditional transfer theory. Part of the problem comes from what I call the "rhetoric of alignment," which is the belief that classroom activities should be as similar as possible to what people do in the target context. Of course, one issue with this perspective is that it's impossible to fully know what students will do in the future - even if we were to teach "professional writing," we could never anticipate how the particularities of different businesses might affect what people write in the workplace. Even more importantly, though, is that this view doesn't account for the importance of time. The world is always in flux, so students will always need to adjust to emergence of new genres, technologies, and social practices. Drawing from Bourdieu and actor-oriented transfer theorists, I argue for a model of practice that views it as a temporal "coupling" between a person's habitus and their social environment. In this view, people are constantly in the process of interpreting their surroundings, adjusting their behavior to accommodate new situations, and selling others on effectiveness of their practices. As Bourdieu puts it, social actors are best seen as "virtuous" that are continually improvising their practices to meet the needs of a changing world. 
The final chapter outlines the implications of this theoretical model for future transfer research. While I suggest some ways that we can revise our methodological approaches to provide a more nuanced understanding of writing practices, my main concern is how we can go about tackling the traditional preoccupations of transfer research from a different perspective. One of the original impetuses behind transfer research is how students deal with new challenges as they move throughout the curriculum. Given that our main task as composition teachers is to prepare students for future writing tasks, we want to know whether students actually use the strategies we teach. However, if we concentrate too narrowly on whether students draw on certain genres, we risk missing how they creatively employ practices learned throughout their entire education. Of course, we need to be careful whenever we pull methodologies from other disciplines, but research into naturalistic decision-making processes offers one potentially useful approach. Studies in this area look at how people go about making decisions in the flow of their activities, where they must deal with time pressures, high stakes, unclear goals, and inadequate information. In particular, their emphasis on how practitioners deal with "unexpected situations" is one strategy that could potentially be used to examine how student navigate new writing tasks. 


\section{CHAPTER II}

\section{THE COMMODIFICATION OF PRACTICE}

This chapter's main purpose is to draw attention to transfer as a concept, as a metaphor that focuses our attention on certain aspects of the learning process. Whenever we invoke a theoretical model, we always draw on a set of metaphorical resources. In this case, they are rooted in the central concept of transfer; however, they emanate outwards from this foundation, making links to neighboring ideas not explicitly under examination. Sometimes, in our eagerness to solve scholarly problems, we forget that we are never bound to a specific concept. We could talk rather convincingly about how students learn without ever referencing the notion of transfer, let alone similar ideas like skill acquisition. For instance, we could imagine the classroom as a struggle, where students must learn to successfully negotiate between clashing cultures. We could see people as learning a "feel for the game," much like a chess player who eventually becomes able to instinctively recognize the best moves. Each of these ideas draws from a different set of metaphorical resources and therefore have their own insights and blind spots. Any examination of transfer must ultimately start with the metaphor itself and what it brings to the discussion.

When we reflect on the process of "transfer," a variety of images immediately come to mind. We might think of a person "transferring" between two different buses. Alternatively, we could visualize money "transferring” between different bank accounts. Or, for those of us in the educational system, we might imagine a student "transferring" 
to a new school. While each of these examples are different in certain respects, they all share two important features. First, what is being transferred is a unified object, a thing that is being literally moved between two locations. Secondly, it is being transferred to a place separated by concrete physical and spatial boundaries. At first glance, this metaphor seems like a strange description of the learning process. After all, when a person learns a practice, it doesn't feel like an object; we can wield a sword, but we can't wield the ability to swing it. In the same fashion, a context doesn't seem like a physical location. If I initially learned how to calculate percentages in math class, I should be able to use this skill to solve physics problems; however, these two tasks have fuzzy boundaries, and it's not entirely clear how to define the difference between these contexts. While the transfer metaphor initially seems to eliminate some vagueness about what happens during the learning process, this clarity is achieved through an analogy to the simpler scenario of transporting objects.

This metaphor might remain attractive to cognitive scientists because of widespread adherence to the computation theory of the brain, which views the mind as little more than a symbolic processing machine that interprets stimuli through a set of complex algorithms. From this perspective, we are born with genetic software that is used to decode the surrounding environment; subsequently, incoming information is "stored" in memory like on a computer chip. For that reason, early chess expertise researchers speculated that masters must have thousands of board configurations stored in their brains to successfully predict their opponents' moves. For scholars operating under this paradigm, the transfer metaphor might have seemed like a compelling explanation of how people adapt skills to new contexts. After all, computers are constructed by humans, so 
the metaphors that explain their operation are drawn from physical objects. When bits of data are recorded on an optical drive drive, this information is characterized as a "file," which is stored in "folders" that can be retrieved at a later date. As such, we can understand why researchers might have been compelled by "transfer"; this process could be easily imagined as the act of copying a "file" to a different "directory." However, recent movements in both social theory and psychology have brought into question this view. In sociology, theorists such as Pierre Bourdieu have argued that while it is tempting to imagine the mind as a rule-driven machine, this seems like an inadequate description of actual social practices. In the realm of education research and psychology, figures like Jean Lave and Etienne Wenger have emphasized the numerous ways that the mind is actively shaped by social factors. Indeed, the emerging consensus is that computational psychology is inadequate for understanding both the brain's neurological structure and the enormous complexity of human cognition.

Given this trend, it is somewhat interesting that composition scholars have only recently begun to draw from transfer studies. While computational models remain an active force in many psychology departments, they have been largely discredited in composition studies. In fact, one of the reasons that scholars turned away from Flower and Hayes model of the composing process was because it represented the mind almost like a symbolic processing machine (Bizzell 233-34). Of course, while the metaphor of the "computer" is a popular way of understanding the brain, psychological theory has a long history of explaining mental phenomenon through appeals to metaphors derived from our daily lives. We also hear educators describe cognitive skills as "tools" that students store in their "toolkits." We talk about people "acquiring" genres, almost like 
they are putting an object in their pocket. We sometimes use the idea of "internalization," which is a metaphor reminiscent of biological research - the brain is seen as absorbing something from the environment, almost like a substance passing through a cell membrane.

The transfer metaphor is appealing partially because it builds on these metaphors that we traditionally use to describe practices. In this view, skills are treated like unitary things, like folders with records on various topics - knowledge, principles, lower-order skills, and other information-which people draw from when handling their daily tasks. When people learn something new about a skill, they are simply adding another record to its pre-existing folder, which they can now draw from whenever they engage in similar tasks in future situations. Therefore, when people "transfer" knowledge to new situations, they are essentially moving records across the various folders in their mental filing cabinet. Essentially, the process is similar to looking through previous records, finding something useful, and moving it to a folder in a different location. David Hammer et al. describe this as a "unitary ontology," the tendency to imagine a "particular piece of knowledge as an intact cognitive unit, in close correspondence with the observable idea or behavior, be it a principle, fact, or procedure" (92). In this perspective, a cognitive skill operates like a discrete entity, a "tool" people use to complete a task.

While Hammer et al.'s terminology is a step in the right direction, it does not explain why it so appealing to depict practices as "intact cognitive units." I want to argue that this tendency stems from some flawed assumptions. Often, we view practices almost like commodities that can be traded in the capitalist economy. In much of our public discourse, we imagine the university almost like a "skills shop" where students can 
purchase the practices needed to become a successful employee in the business world. My term for this perspective will be commodification. By invoking this concept, I'm echoing the language of Allan Luke, who argues that genre scholars tend to view power in this fashion. He claims that "in the current educational economy most contemporary pedagogies tend to reify power, to turn power into an object in the world which can be commoditised, containerised, and, quite literally, bought and sold on an educational marketplace" (322). I'm also drawing from the work of Bruce Horner, who argues in Terms of Work for Composition: A Materialist Critique that our focus on educational outcomes sometimes results in treating "students, their skills, or their consciousness as commodities" (18). Central to the process of commodification is the transformation of practices into marketable things. While this word is often employed loosely in everyday discourse, I will use it to refer to something specific—namely, an entity that is bound together by a kind of internal glue, whether it be the laws of Newtonian physics, the rules of logic, or some other mysterious phenomenon. When researchers view practices in this way, they are treated almost like unitary objects that will remain unchanged unless somehow disrupted by an external force.

To avoid this problematic tendency to imagine practices as commodities, my recommendation is that we search for concepts that draw on a different set of metaphorical resources. Just to clarify, my emphasis on de-commoditizing the mind should not be confused with a desire to de-metaphorize it. Indeed, it is impossible to even conceptualize a phenomenon without drawing a metaphorical comparison, whether it be to physical objects or some other type of entity. My goal here is simply to demonstrate the myriad of ways that writing skills are commoditized in composition research. 
Sometimes, this process is overt, such as when Russell directly compares genres to physical tools. However, in some instances, commodification can be more subtle, such as the numerous cases of scholars portraying discourse communities and contexts with clearly bounded locations. As I hope to demonstrate, commodification is prevalent in certain strands of composition research, despite the numerous attempts to undermine such tendencies.

\section{Practices as Unitary Things}

Compositions scholars frequently examine writing practices through the lens of genre theory, which has its origins in literary criticism, rhetorical studies, and linguistics. While researchers have certainly utilized other approaches, I want to focus on this model because of its historical importance to transfer research. Many studies have been directly formulated in terms of genre (Freedman; Wardle; Artemeva). In addition, many researchers have claimed that genres are best learned within their target context, which is part of the reason we must be skeptical about the possibility of transferring skills from writing courses to other environments (Russell; Freedman). Finally, while genre theorists have not always directly invoked the transfer concept, their work often explores similar issues; in fact, the recent interest in transfer studies stems directly from problems originally formulated by genre theorists, especially Freedman's arguments against explicit teaching and Russell's "activity system" approach. At the moment, genre theory is the dominant model of writing practices among transfer researchers in our discipline.

When rhetoricians trace the history of North American genre theory, they often begin by highlighting the importance of Carolyn Miller's "Genre as Social Action," which first articulated the seminal notion that genres are not linguistic forms but rather 
typified responses to recurring situations. Central to her theory is the notion of an exigence, which is a situation that invites a certain kind of action. Over time, certain exigences have a tendency to reoccur, which results in the development of consistent, institutionalized textual responses by members of a particular group. While several motivations likely drove Carolyn Miller's work, one of her primary concerns was overcoming the view that genres are just rigid textual forms. As she argues, a "rhetorically sound definition of genre must be centered not on the substance or the form of the discourse but on the action it is used to accomplish" (151). Or, at another point, she claims that "a genre is not just a pattern of forms or even a method of achieving our own ends. We learn, more importantly, what ends we may have" (165). She believed that equating genres with forms was one of the main problems that plagued traditional genre theory.

As a result of this aspect of Miller's work, contemporary scholarship is filled with denunciations of any effort to equate "genres" with "forms." Medway argues that while "genres are still expected to display characteristic textual forms . . identifying patterns of text format, syntactical and lexical choice, and discursive ordering, however, is no longer considered sufficient for pinning down the genre" (123). In Writing Genres, Devitt argues, "form alone cannot define genre. Theoretically, equating genre with form is tenable only within a container model of meaning, for it requires a separation of generic form from a particular text's content" (9-10). In "Rethinking Genre in School and Society: An Activity Theory Analysis," Russell argues that "we must go beyond the conventional notion of genre as a set of formally definable text features that certain texts have in common across various contexts" (513). While genre theory has helped combat 
the notion that writing involves just following a set of formulas, the problem is that researchers have tended to focus narrowly on a particular metaphor-in the case, the tendency to equate writing practices with forms - even though this is just one example of how researchers commidify writing practices. In reality, the difficulty is with an entire class of metaphors that reduces writing practices to unified things.

A metaphor that reduces writing practices to commodities ultimately runs into three problems. First, it has trouble explaining the mental overlap between practices. While we are accustomed to viewing skills as stored in isolated containers, this view has difficulty explaining how learners build on previous practices and adapt them to new contexts. Practices must somehow share mental resources-otherwise, each time we faced a new learning situation, we would be forced to start with a blank slate. Secondly, these metaphors also create the problem of location. Obviously, if practices operate like commodities, we must have some sort of process for acquiring them. However, experienced practitioners are not simply able to dump the contents of their minds directly into future generations. Interactions between individuals always must be filtered through language, which rules out the possibility of ever achieving the perfect transmission. For that reason, social theorists have historically resorted to positing the existence of transcendental entities — such as social structures and the collective conscious - that learners must internalize. The idea is basically that practices are downloaded from some kind of mystical database. Of course, people are not literally connected by cables to a computerized network, so the question arises of where we might find these mysterious transcendental objects. Finally, a potential metaphor must also deal with the problem of change. When we visualize most commodities, we imagine them as only slightly 
changing with the passing of time. A hammer might achieve some wear from frequent use, but it still essentially remains the same object. In contrast, humans are inextricably embedded in the flow of history. We are thrown into a world of pre-existing social relations, where people already have an investment in certain practices and therefore encourage their development in future generations. Our interactions in this environment leave traces on our mind and influence our future actions. We also continually face new situations, which subtly shape our current perceptions and sometimes radically transform our practices.

Unfortunately, while the transfer literature is filled with example of scholars dismissing the idea that genres can be reduced to textual forms, many theorists invoke different metaphors that still commidify practices in various ways. David Russell's work is perhaps the most prominent example of this issue. In his numerous attempts to inject activity theory into genre studies, he has drawn from a variety of metaphors. While he sometimes compares to practices to games, he more commonly refers to genres as "tools" that people use to accomplish various purposes. In "Activity Theory and its Implications for Writing Instruction," he describes activity systems as "goal-directed, historically situated, cooperative interactions," providing the examples of a "child's attempt to reach an out-of-reach toy, a job interview, a date, a social club, a classroom, a discipline, a profession, an institution, [or] a political movement" (53). He argues that activity systems have three components: (1) subjects, which range from individuals to social groups, (2) objectives that the subjects strive to achieve, and (3) "mediational means," tools that allow members of the activity system use to accomplish their objectives. These tools include physical objects, such as shovels, and semiotic ones, such as written genres. 
Members of an activity system might accomplish their objectives through the use of several tools - for example, if a pothole exists in a road, a construction worker might personally fill it with a machine, or he/she might choose to fill out a work order. These tools are defined by the activity system, to the extent that plumbers and carpenters could be described as using fundamentally different "hammers."

As Amy Devitt argues in Writing Genres, Russell's work supports two possibilities for genre's role in an activity system. At one point, Russell defines genre as "variance in semiotic tools according to the activity," which seems to indicate that genres are not exactly tools but how tools are used in activity systems (54). At other times, Russell equates genres with tools, such as when he argues that "using a certain genre of writing (a certain kind of semiotic tool) as part of an activity system is like using the gearshift of a car (a certain kind of mechanical tool) as part of [an] activity system" (70). The problematic thread that unites these two interpretations is the involvement of tools. As Devitt explains, the problem is that once genre is associated with semiotic tools, it is no longer conceived primarily "as an action ... [which] diminishes the role of people in creating and using genres" (47). In addition, imagining genre as a tool "limit[s] the nature of genre to formal formulae, a preexisting, static, material object that people can pick up and use or just as easily set aside" (48). While viewing genres as the routinized use of tools escapes this dilemma, the problem is that a person's actions are still bounded by the constraints of the semiotic tool. Two subjects might use a hammer in a different way, but we still call it a "hammer" because it has physical features that remain stable outside of a particular activity system. 
This metaphor produces all three of the problems that often arise from commidifying practices. First, it becomes difficult to imagine the mental overlap between different skills, mainly because of the comparison to material tools. While a hammer and a screwdriver might share some features on an abstract, conceptual level, they remain discrete physical objects, which are stored in different compartments of a person's mental toolkit. The theory fails to account for how writing a personal statement might involve some of the mental components that are used in other genres, such as non-fiction narratives. Secondly, this view of semiotic tools introduces the problem of location. The reason that mechanical tools can be acquired is because they are material objects. They can be purchased from department stores, borrowed from friends, and constructed at factories. Semiotic tools are not objects with the same properties; therefore, acquisition only occurs on the metaphorical level. In that sense, acquisition-and other similar verbs, such as internalization - are misleading ways of describing learning, which involves a complex process of modifying old skills and developing new ones. Thirdly, this view of genre introduces the problem of change, particularly as it relates to how learners might modify practices for use in future contexts. Imagine a novice homeowner that only owns a shovel. If he/she is faced with the need to fix a staircase, he/she has limited options for completing this task. The homeowner could attempt to use the shovel to drive nails into the wood, but this solution is not ideal; for him/her to accomplish his/her objectives, the best strategy is to acquire more suitable tools. Unless a new situation requires the same tool, it is difficult to imagine how a learner would build on previously acquired practices to accomplish a new set of objectives. 
Of course, many theorists explicitly avoid reducing genres to "tools," although such language remains popular among many scholars. In fact, even Russell seems to recognize the limitations of this approach, which is the reason that he emphasizes that genres are "typified ways of interacting with tools" (408). Viewed in this way, genres become more like the habitual way of using a tool in an activity system. This solves the problem of location, for habits are acquired through observing the behavior of other people; for example, we can watch how an expert carpenter grips a saw, ask him/her for some general advice, and then practice it ourselves with his/her guidance. We also have somewhat solved the problem of overlap—if I learn the genre of "saw carpentry," aspects of this genre might help me use tools in other activity systems; for example, if an expert carpenter tells me, "always remember to let the saw do the work," I might employ this technique to help me avoid applying too much force when hammering a nail. However, this conception of genre derives its explanatory power by an appeal to a different concept —namely, that of habituation. In fact, this theory gains its strength by explicitly diminishing the theoretical function of "tool." Indeed, it is difficult to fully understand the role played by "semiotic tools" in this framework. The difficulty is that it is not problematic to talk about habitual ways of using physical tools because they are material objects. While they might be used differently depending on the activity system, their material form will remain constant regardless of the circumstances. In contrast, a semiotic tool has no such material existence. As such, once we begin viewing "genres" as "habitual ways of using semiotic tools," the identity of the "tools" becomes a mystery. The only recourse seems to be depicting genres as textual forms. However, the whole point of contemporary genre theory is to move beyond such problematic comparisons. 
Interestingly, even though Devitt spends considerable time developing a similar critique of Russell's work, she likewise commodifies writing practices in her equation of genres to "cultural software." What makes this move fascinating is her deliberate effort throughout Writing Genres to avoid reducing genres to unified entities; she continually resists the attempt to reduce genres to forms, which actually serves as her primary rationale for questioning Russell's approach. In Devitt's case, she appeals to the notion of "cultural software" in her explanation of how genres change over time. In her mind, one of the problems with existing rhetorical theory is the tendency to see genres as either allowing for certain textual choices or inhibiting a writer's freedom. As she explains, we often associate generic constraint with slavery, as if any restrictions placed on the writer is somehow equivalent to their minds being shackled by the chains of tradition. However, communication would not be possible without some kind of standard. For two people to successfully communicate, they must be utilizing a similar set of linguistic practices. On the other hand, while some convergence must exist among speakers, there also must be divergence; in any effort to produce an utterance that meets the needs of a particular rhetorical situation, we must come up with a novel combination of words, phrases, and other elements. As she explains, "when I write the sentence I am writing now, I am writing a sentence I have never written before ... in fact, all of the sentences in this paragraph, all of our nonroutine utterances, are new and original in some basic way" (140). In this view, we are always drawing on our existing generic resources and weaving them together into innovative patterns. Therefore, constraint and choice should not be viewed as opposing forces; instead, we should imagine them as complementary impulses: "Janus-like, genres inevitably look both ways at once, encompassing convergence and 
divergence, similarity and difference, standardization and variation, constraint and creativity" (162). Her main objective is to demonstrate that some standards are necessary for the creative process. In addition, these constraints have ideological origins, which means genres are not politically innocent and serve the interests of certain cultural groups.

The problem arises from her explanation of the origin of these generic resources, which she explains by invoking J. M. Balkin's notion of "cultural software.” While his original theory likely holds numerous complexities, my goal is not necessarily to challenge his work but rather how Devitt utilizes it. Much like Russell, she demonstrates a certain amount of ambivalence about her metaphor, but she justifies its use by explaining that Balkin "treats cultural information as tools for human cognition, ways of understanding the world, without which humans would be unable to make meaning" (138). Later, she goes on to say that "genres, as part of cultural information or 'software,' helps humans understand their experience, as created and transmitted by other humans, and preserve stability while allowing change and creativity" (138). It is interesting that her argument makes use of the same tool metaphor that she critiques in Russell's work; however, the more important issue is the metaphorical resources invoked by the notion of "cultural software." One advantage of the term is that it immediately makes concrete how people acquire textual practices; in this model, writers are viewed as downloading genres from a vast cultural network. It also provides an explanation for the rule-like appearance of our linguistic interactions. "Programs" are nothing more than a sequence of logical rules for dealing with incoming data, which means that to explain the convergence 
between how people respond to similar rhetorical situations, we might compare them to two computers executing the same software.

Of course, this metaphor introduces similar problems to those created by the notion of tools. To a limited extent, it avoids the problem of overlap between genres, for programs often draw from similar resources—on a basic level, they all utilize the structure created by the operating system. In addition, they sometimes draw on a common set of software for certain functions, including various movie players, internet browsers, graphical drivers, and compression algorithms. However, it is an open question about whether we feel this way while actually using programs. When we open up some word processing software on the screen, we often imagine its existence as distinct from our internet browser, even though both programs often share many resources. In addition, we see ourselves as copying these programs wholesale between different locations; we "download" programs from the internet, and we then "copy" files onto our hard drive. However, the problem of location becomes even more prominent with this particular metaphor. While software is often downloaded from a network of computers, human are not connected by a set of internet cables. We also don't have optical drives for storing information. The temptation is to argue that the programs are stored in a transcendent metaphysical location, but she explicitly rejects such a move; as she points out, "such cultural software is created and transmitted by human beings; it does not exist as some suprastructure or system independent of the people using it" (158). Therefore, the immediate question is how we might obtain such software.

Her appeal once again is to a vague metaphor that operates as a shortcut for the learning process. While Russell's preferred term was acquisition, she instead draws from 
the notion of transmission. However, this concept immediately introduces the limits of the idea of cultural software. When a computer transmits a piece of data to another location, this movement ultimately involves transporting a file wholesale between contexts; if a person copies a text document onto a flash drive, he/she should be able to open the exact same file at a different location. As a result, this metaphor seems to indicate that two people will have identical textual practices. However, as Devitt continually emphasizes, genres often feature tremendous variation, to the extent that each individual linguistic performance is to a certain extent a completely novel utterance. The problem is that software never deviates from its programmed capabilities. In contrast, people are continually diverging from expectations and blurring practices into novel forms.

To resolve these difficulties, Devitt introduces the distinction between perfect and imperfect transmission. As she explains, "what keeps such ideological power from overwhelming the benefits of genres ... is that genres are still created and transmitted by individuals, by human beings with all their imperfections, including the imperfect transmission of ideology" (161). Therefore, a genre is never "replicated exactly the same in each individual" (161). Unfortunately, this proposed solution generates two new problems. First, if we frame the transmission as an imperfect one, then we implicitly assign a set of objectives to the learning process - namely, the ideal of perfect transmission, which is the exact reproduction of a cultural practice in another individual. Secondly, the notion of imperfect transmission is strained on a metaphorical level. When programs are copied between two computers, the transmission necessarily involves the duplication of identical programs. In fact, when the transmission is imperfect, the files 
become corrupted; what is created is not an exciting new program but rather a useless piece of digital trash. Once again, my point is not that we need to abandon metaphors for understanding practices. Indeed, it is impossible to conceptualize the mind without making such concrete comparisons. However, we need to resist metaphors that equate learning with transporting objects between locations. Practices are not things that are transmitted, copied, or internalized by learners.

Unfortunately, programs are not the only metaphor that depicts the mind as operating through a set of rules. Many researchers have also compared writing skills to games, which is appealing partially because they are defined by "rules" that restrict the player's possible actions. The most prominent use of this metaphor is Russell's ball game analogy, which has proved especially convincing to transfer researchers (Smit; Downs and Wardle; Bergmann and Zepernick). To quote Smit's summary of the analogy, "the ability to use a ball is a particular skill to be used in a particular manner in a particular context, and we would expect the skill of being able to use a ball in a certain way to be transferred only to other similar uses" (120). In the same way, writing in a genre requires learning how to play the "game" of an activity system, and no guarantee exists the skills learned in one context will necessarily apply to future ones. A practice will only "transfer" when the situation is similar enough to draw upon the same cognitive resources.

Let me begin by distinguish between two different ways of comparing games and writing practices. First, we can conceive of writing as like playing a game. In many respects, the metaphor gains its persuasiveness because of our sense that writing is often a game-like practice-i.e, it contains the same mixture of seriousness and playfulness that 
characterize most sports. When we are putting together a resume, we talk about "gaming the system," as if the task is to predict the audience's reactions and somehow bamboozle them. Secondly, it also accurately captures the temporal element of textual practices. If a colleague passes along a memo that suggests the company might eliminate our department, this action will set in motion a series of textual responses, some which might be tactical attempts to position ourselves in such a way that we will avoid being laid off. Anne Freadman accurately captures this strategic element of writing in "Anyone for Tennis?," which she begins by comparing writing to players exchanging tennis balls. Indeed, if we imagine writing as like playing a game, the comparison illuminates a great deal about the writing process, especially how it always involves the act of responding to other people.

Alternatively, writing can also be imagined as a game. Much like games are defined by a set of constitutive rules, genres can be seen as distinguished by the same type of rigid boundaries. When scholars use the metaphor in this fashion, writers are imagined as carrying a set of rules in their heads that determine how they engage in textual practices. Of course, if people want to successfully communicate, they must be equipped with the same rules; otherwise, they will be playing different games, much like two chess players who fail to agree on the movement of the game pieces. As Freadman points out, the difficulty with this metaphor is that "it suggests that once you have learnt the rules ... the playing of a correct game following automatically, like the output of a simple algorithmic programme in a computer." She argues that certain ways of framing the metaphor make writing seem like nothing more than following a recipe (46). As she emphasizes, we must avoid confusing understanding how to play a game with its rules. 
Obviously, while many people are capable of regurgitating chess rules, only a fraction them know how to actually play; the rules are at best a set of training wheels used during the learning process. In addition to knowing the rules, people must develop a "feel for the game.” They must learn to interpret the broader strategies used by their opponents. In addition, they need to be capable of instinctively respond to these moves by employing an assortment of tactics. As Freadman argues, the rules only determine the range of possible actions. The actual play is determined by other factors, such as the player's previous experiences and his/her cultural background.

Interestingly, even though Freadman notes these difficulties, she remains unable to move beyond the idea that people carry around a set of rules in their heads that shapes their social practices. In fact, her essay could be viewed as an effort to somehow rehabilitate the notion of rules. To help explain the variety of behaviors that constitute playing the game, she introduces the notion of a ceremonial, which she defines as "games that situate other games: they are the rules for the setting of a game, for constituting participants as players in that game, for placing and timing it in relation with other places and times" (47). At another point, she explains that ceremonies involve "the warm-up, the toss, and, at the end, the declaration of the winner and closing down rituals - showers, presentation, or the drink at the bar" (46). Her argument seems to be that the game is not just determined by its rules; it also is shaped by its surrounding cultural rituals. To give a simple example, polo and lacrosse would likely encompass much more indecorous strategies if they were not perceived as genteel sports. In addition, poker might not involve the same degree of deception and bluffing if it were not seen as housed in working-class bars. Unfortunately, the ceremony metaphor fails to overcome the larger 
problem, mainly because it simply adds a new layer of rules. As she points out, ceremonies are "the rules for the playing of a game, but they are not the rules of the game" (47). In fact, as she continually emphasizes, they can essentially be imagined as a different type of game; she only chose to retain both terms for "tactical purposes" (46). The question then naturally becomes whether ceremonials also operate like "recipes." Obviously, if people conduct rituals simply by executing the rules in their heads, then it is not clear how Freadman avoids reducing people to rule-driven machines.

We might be tempted to argue that ceremonies themselves are also embedded in other games - as I pointed out, lacrosse is often seen as a genteel sport, while poker is viewed as having working-class origins. We could say that people have learned to play a wide range of games, which have been partially developed from their race, sexuality, class, and gender backgrounds. In this view, the only reason the "game" metaphor seems machine-like is because we only interact with a limited number of tightly bounded games, while the human mind in fact contains the rules for thousands of games that it executes for various reasons depending on the circumstances. The issue then becomes one of complexity. The idea is that if we could stack up an enormous number of rules, we would have a worthy model for how the mind operates in daily life. In fact, computer scientists originally took this approach in artificial intelligence research, and their efforts have only been successful within certain highly restricted domains, such as a playing chess and moving blocks. As Hubert L. Dreyfus and Stuart E. Dreyfus explain, the thought was that the computer only needed specific facts and a set of rules for determining the appropriate action. However, researchers quickly discovered that minds don't operate through such internal recipes. Imagine that you wanted to construct a 
computer that could drive a vehicle. To design such a program, one would presumably start by downloading an encyclopedia of traffic knowledge into a storage device.

Unfortunately, this would not be enough, for the machine would not know how to process such information; therefore, one would also need to include a set of rules for calling upon the facts necessary for a certain situation. One might instruct the computer to stay within the traffic lines, and this would be reasonable for most situations - but once again, this would not be sufficient, for exceptions always exist to all seemingly universal rules. A car might veer into the lane, a pedestrian might suddenly walk across the street, or the lines might disappear in a traffic zone. As a result, one needs a set of rules for dealing with the exceptions to the normal rules; then, one needs a set of rules for determining exceptions to these exceptions. In other words, the mind seems to behave "as if" following rules, but when examined in its full complexity, it appears to have an almost infinite capacity for adapting to new situations.

The reason that the game metaphor initially seems compelling is because it convincingly captures the mixture of constraint and possibility that seems to characterize our lives. Games have two properties that make them an appealing model for understanding social practices. First, a game's rules construct a space for a set of actions that otherwise would not exist. If someone had never devised the game of chess, we would have never even considered moving about pieces in such peculiar ways. But the rules also define the game's limits-players could not start deciding to arbitrarily move pieces in a different fashion, for the game would ultimately become meaningless. In the same way, our textual practices also seem to have these features. If we are writing a business memo, we probably shouldn't begin with a prose-poem, unless we have some 
compelling reason to deviate from the standard approach. To put this another way, the metaphor seems to explain why people don't engage in certain behaviors; we can stipulate that the reason someone would never leave the house naked is because he/she has a tacit "rule" stating that nudity is only appropriate in certain situations. The problem with this perspective is that it imagines the mind as a device of infinite possibility that is "restrained" by culture. At one point, Freadman argues that "ceremonial rules" are perhaps viewed more like etiquette. In her opinion, this new metaphor is useful because it helps us resist viewing rules as fixed laws; rather, they operate more like "good manners," which we recognize as somewhat arbitrary and continually renegotiated. However, she insists that even this view is a fiction, which usually operates "in a group that thinks of itself as unregulated by anything but spontaneity and fellow-feeling. Such rules, like the rules of genre or a ceremony, are there "to make things work"' (58). The idea here is that without some kind of internalized rules, our behavior would essentially be open to any possibility. But the mind is not an infinitely powerful organ. From the moment we leave the womb, our genetic faculties are shaped by culture. No source of spontaneous free will exists inside our flesh that must be restrained; we are always already open to only a limited range of actions.

Like the other attempts at commodification, the problem with the game metaphor is that it begins with a unified object and then attempts to transform it into some kind of "tacit" mental entity. The problem is that once we start to examine cognitive skills, they don't seem like the rule-governed behaviors that are suggested by the comparison. When we are referring to a game, we are talking about an activity governed by explicit rules, ones that can be easily recalled as knowable facts. When we claim that a person "knows 
the rules of football," it does not make sense that they only have tacit knowledge; if he/she was unable to immediately tell us that a touchdown is worth six points, we would probably start to doubt his/her knowledge of the sport. The rules must be explicit for them to define the game's limits. Otherwise, they would not help resolve in-game disputes. In contrast, most writing practices seem like they are not governed by such rules. At best, some might be regularized enough that they seem "as if" they follow rules; however, even in these rare cases, the basic patterns often disguise a substantial amount of variation. Even if we limit our discussion to actual games, the logic that defines them mostly by their rules seems questionable. They might be a game's most recognizable feature, but they do little more than establish the range of possible actions. While the objective of football is to move the ball into the end zone, this "rule" says little about the actual play, which is informed by an entire history of strategies that is being continually refined by players. The "rules" also say little about the game's place in the larger culture; surely, football would be a different sport if it had not become a national pastime and come under the management of wealthy elites. It would also be much different if separated from its working class roots. Indeed, to be fully understood, a game must be imagined as a social practice, which seems incapable of being reduced to a set of rules.

These three metaphors are only some examples of commodification. We have a long history of using similar terms, including verbs that suggest students "internalize" skills or comparisons that make writing seem like a theory guided by tacit "assumptions." The problem is that all these metaphors make skills seem like things held together by some kind of internal glue, so that when we try to imagine how a student would "transfer" a practice, we become convinced that it must be moved wholesale between 
contexts - to put this in the language of one of the metaphors, we have trouble imagining how students might utilize their previous skills to play a game with different rules. If we want to develop a more accurate view of how students learn, we need a more powerful model. After all, writers are continually moving between rhetorical situations, and they seem perfectly capable of drawing on their experiences to accomplish new tasks.

\section{Contexts as Bounded Places}

The previous section focused on the tendency among genre theorists to represent practices as commodities, which people internalize through their involvement with certain social groups. But this discussion only deals with part of the transfer metaphor. If we once again visualize the process of transfer, we will recall that it involved transporting a practice between two contexts, which are frequently imagined almost like places in the material world. While the term place is employed loosely in our daily speech, I will use it to refer to a space with clearly marked boundaries. Even when the concept is applied to physical locations, it is merely as a convenient fiction that enables the commodification of space. If material space was viewed as a common resource shared by all biological creatures, we could not restrict access to spaces and sell them in the marketplace. Given that we spend our lives moving between such commodified places, it is relatively easy to view our social world in this way; however, as many scholars have pointed out over the last couple decades, viewing contexts like places creates a number of problems.

One formulation of this view that has been criticized by many scholars is the notion of discourse communities. In this view, what creates intelligibility among certain people, whether they are scientists, rural farm workers, or politicians, is a shared discourse, a common way of describing and thinking about the world. The danger with 
the metaphor is that it sometimes leads scholars to adopt a monolithic picture of social groups. Many “discourse community” models suffer from what Paul Prior describes as “structuralist" tendencies, where language communities are viewed as "discrete, autonomous territories with core regions constructed from common language, knowledge, values, and rules" (18). As Marilyn Cooper observes, the concept can be seen as implying that "features such as shared values, conventions of language, and norms of behavior" are nothing more than "static standards that are used to determine who is and who is not a member of the community" (204). In "The Idea of Community in the Study of Writing," Joseph Harris suggests that instead of seeing academic work as taking place in a "single cohesive community," we should instead use the metaphor of the city, which captures the sense of a place consisting of multiple, competing languages (20). The problem is that discourse community models only satisfyingly explain two phenomenon: (1) the cohesion obtained between the practices of a group's members and (2) how they differentiate themselves from participants of other groups. Unfortunately, the metaphor is less effective at explaining that heterogeneity and historicity of communities, whose members are embedded in shifting power relationships and are continually struggling to define the group's identity.

Perhaps the most striking example of these problems in the transfer literature is Anne Beaufort's work, whose recent longitudinal research examines the development of a single student as he moves throughout the curriculum. To offer a detailed picture of his writing practices, her data comes from a wide range of sources, including students interviews, writing samples, evaluators' comments, classroom observations, and various course materials. Her analysis is framed via a model of writing expertise that was 
developed in her previous work. She imagines writing practices as composed of five overlapping components, including discourse community knowledge, genre knowledge, writing process knowledge, rhetorical knowledge, and subject matter knowledge. The largest of these domains is discourse community knowledge, which she visually depicts as a large circle that encompasses the other four aspects of writing expertise. As she argues, discourse communities are "based on a set of shared goals and values and certain material/physical conditions ... [and] establish norms for genres that may be unique to the communities" (19). At the outset, I want to acknowledge that she acknowledges that "discourse community" is a contested concept. However, while she recognizes the difficulties with the metaphor, she still has a tendency to represent contexts as places with clearly marked boundaries. ${ }^{1}$ She frequently fails to acknowledge the heterogeneity that can exist within a single classroom.

For example, she rarely comments about how her research subject's ideological commitments might have influenced his development. In fact, we discover little about the student's background, aside from some interesting moments in the interviews. At one point, we discover that he is religious, which directly influenced his decision to pursue certain essay topics. As he explains, "I'm a Christian, so that's kind of interesting to see

\footnotetext{
${ }^{1}$ At one point, Beaufort seems to anticipate my critique of her work. As she explains, some people might accuse her of viewing her categories as "stable" entities; however, people of this "philosophical bent" confuse a model that depicts writing practices as "distinct yet overlapping categories" with one that imagines "those categories are fixed and discreet" (21). Later, she claims that her goal is "not to win some philosophical argument about the nature of truth" but "to try to get a more fine-grained and unified sense of what is going on when we study student behaviors and a broader view of what we should consider in creating writing curricula" (22). I agree that a model should not be criticized as "essentialist" simply because it deploys categories. In fact, I would argue that ultimately we need to categorize a phenomenon in order to operationalize a theoretical model. Once again, the problem is not with categories but with metaphors that guide our thinking in problematic ways. As I hope to demonstrate, she seems to conceptualize discourse communities as clearly marked places once she actually uses her framework to interpret the data.
} 
my own kind of heritage and kind of how ... I guess it helps personally understand what can go wrong in a church" (75). This aspect of his identity directly impacted how he imagined historical discourse. He often felt spiritual subjects were excluded from the scholarly conversation: "In some respects I still feel like it's outside the realm of history . . . well, we don't look at the supernatural. We don't deal with this here, okay?" (75). In addition, this focus caused him to avoid certain courses, which resulted in his exposure to only a limited range of historical writing practices In particular, he avoided courses on "the race/gender theme" because it seemed too "all-consuming" (75). Therefore, while we might loosely talk about the "context" of a history course, we must acknowledge that individual students will largely operate in different "contexts" depending on their previous experiences. In this case, the student's religious background directly influenced what he learned from the curriculum. His writing development was also likely affected by his race, class, gender, and sexual orientation, which partially explains his aversion to particular courses. His education might have been impacted by a variety of other factors, such as his relationship with other students and his perceptions of individual teachers. Partially because of how university classrooms are being conceptualized, many of these elements are rendered invisible.

While her research is more sensitive to the fractured nature of academic disciplines, she rarely acknowledges that part of the learning process is learning how to navigate these conflicts. Instead, she seems to imagine the student's task as learning a set of clearly defined writing practices, such as beginning an essay with a concise thesis statement. Throughout her discussion of the student's transition from freshmen composition, we get little sense of how the student coped with the different conceptions 
of "writing" that he faced in his history courses. Interestingly, the student highlights many of these complexities in his interviews. As I previously mentioned, he was aware of the "race/gender" courses, which promoted a certain "line of thought and analysis" (75). He also talks about a professor who considered himself as a "futurist," which meant that he used historical facts to make predictions about future events. In contrast, he also knew a professor who dealt entirely with past events and believed history was mostly unpredictable (74-75). Each of these different perspectives makes a claim about what is valued in a historical interpretation. As Beaufort herself notes, the features of historical genres are difficult to discern because no standard exists of "what 'counts' as historical proof, i.e. what precise types of warrants and claims constitute the rhetorical features of an argument in history" (71). As the student moved between classes, he was likely confronted with a dizzying array of writing practices. Part of "learning to write" involves making a decision about where one stands on such issues. Students must become acquainted enough with the range of practices that exist in a particular group to know how to effectively position themselves within this conflicting web of relationships.

These problems are compounded by her desire to classify transfer as either "positive" or "negative." Many transfer researchers seem motivated by a desire to smooth over the fractures in the curriculum, which can be seen by the fact that many studies involve simply cataloging the disjunctions that students experience when writing in different contexts. In Beaufort's description of the struggle that her student faced when writing for his history courses, she describes numerous instances of "negative transfer" where he "inappropriately" applied strategies from first-year composition. At one point, she claims that "the genre of the "essay' in freshmen writing was characterized largely as 
a loose exploration of a topic, whereas in history, for the most part, [his] professors wanted focused, linear arguments" (104). In addition, historical essays focused "on textual analysis or comparisons of sources from 'objective' points of view, whereas in [his] freshmen writing classes the emphasis was on self-exploration and connecting personal interest to wider social issues" (104). The suggestion is that this "disjuncture" ultimately hampered the student's development. However, his actual statements seem to indicate that his first-year writing course provided him with a framework for resisting some of the writing practices in his history courses. He directly states that he "sometimes value[s] the flow in the writing over the step-by-step kind of point-by-point analysis" (90). In fact, he argues, "So people will be able to say, yeah, well, what about this? Then I'd have an answer for that, but I don't want to bring it up myself because it gets in my way ... valuing the rhetoric, you know, over the substance" (90). While we might not necessarily agree with his rationale, this statement reveals a writer who is aware of the relevant discourse conventions but who has chosen another path for ideological reasons. His deliberate attempt to position himself in a certain way demonstrates a student who is starting to learn that communities are not homogenous entities and in fact tolerate multiple conflicting practices.

Unfortunately, the tendency to label any kind of struggle as evidence of flaws in the curriculum is a regular theme in transfer research. In "A Stranger in Strange Lands: A College Student Writing across the Curriculum," Lucille McCarthy describes the results of a similar study that looks at a single student as he moves between his courses; primarily, she is focused on how his writing developed as he transitioned between freshmen English, biology, and poetry. In addition to conducting interviews and 
classroom observations, she also utilized think-aloud protocols, where he was asked to produce a first draft of an essay for one of the three courses. Interestingly, he is also a white male student who ends up studying a scientific discipline. Perhaps not coincidentally, even though her data was collected almost two decades prior at a different institution, her findings echo many of Beaufort's conclusions. As she argues, "although the writing in the three classes were in many ways similar, [the student] interpreted them as being totally different from each other and totally different from anything he had done before" (243). She painstakingly documents his numerous difficulties. In his poetry class, his critical essays were basically summaries, primarily because he discussed each stanza in sequential order rather than use a thesis-driven structure. He utilized the same approach when analyzing a peer-reviewed journal article for his biology course. In the think-aloud protocols, the student seemed driven by the considerations of each individual assignment, rarely reflecting back on previously learned practices. The learning process seemed to "start over" each time he was faced with a new situation.

To help explain this phenomenon, McCarthy directly invokes spatial metaphors, inviting her readers to imagine the student like "a stranger in strange lands" whose "journey from one discipline to another" closely resembled the experience of "a newcomer in a foreign country" (234). As she further elaborates in her conclusion, "the courses for writing may be so different from one classroom to another, the ways of speaking in them so diverse, the social meanings of writing and the interaction patterns so different, that the courses may be for the student writer like so many foreign countries" (260). Building on this analogy, she compares disciplinary practices to new languages, claiming that her study "raises questions about how teachers can best help student 
'strangers' to become competent users of the new language in their academic territory" (262). Once again, these metaphors are largely appealing because they compare disciplines to contemporary nation-states, which are politically defined by rigid spatial boundaries. She imagines students almost like courageous explorers who must "learn the language" of the native population to survive. This conception offers little room for resistance, mainly because students are viewed as needing to successfully "adapt" to each new disciplinary context; otherwise, they will simply perish in the wilderness of academia. Finally, even if we confine our investigation to a single classroom, her argument fails to account for the fact that the "native language" of a discipline contains several conflicting discursive practices. To a certain degree, the "stranger" metaphor is useful because it moves beyond the notion of writing as an acultural practice. Potentially, the metaphor could be reformulated by looking at postcolonial theories of nation-states, which often acknowledge the fuzziness of both borders and linguistic practices. However, in its current incarnation, the study treats contexts mostly as bounded locations.

When we imagine learning to write as essentially the process of adopting the "native language" of a place's residents, the problem is that struggle always seems to be a sign of maladaptation. The idea is that a person's previous linguistic practices are somehow "interfering" with those of the new community. The implication is that we need to work toward alleviating such conflicts, which is part of the reason that transfer research is so focused on simply cataloging the challenges that students experience as they transition between classes. To justify his reform proposals, Smit cites Walvoord and McCarthy's case studies of four different disciplinary courses, pointing out the numerous examples "of students who try to apply knowledge and experience that is inappropriate or 
in ways that are not helpful" (129). In Patrick Dias et al.'s examination of the transition between university and workplace contexts, they argue that new workers often report "feelings of disjuncture and anxiety quite different from those experienced in their schooling" (196-7). They argue that these struggles ultimately stem from "the need to learn new ways to learn [work] genres" (197). In Randall Popken's theoretical discussion of transfer issues, he distinguishes between "positive" and "negative" transfer, arguing the latter category includes cases that "create discord: the properties of the familiar genre do not match those of the unfamiliar" (6). Borrowing from the language of Mary Ann Eiler, he characterizes such situations as "genre interference," when "properties of one genre are inappropriate or dysfunctional when used in another context" (6). Rather than seeing these struggles as constructive attempts to draw on existing resources, these researchers portray students as simply failing to successfully utilize their previously learned skills. To put this in the language of commodification, students are viewed as consumers who have purchased the wrong tool to solve a particular problem, almost like a mechanic who brings a hacksaw to remove the tire from a vehicle. The idea is that only one "tool" will fit the needs of the situation. As I have pointed out throughout this section, the problem with this view is that any given community is actually composed of people engaged in a variety of discursive practices. An adequate theoretical model must take into account that social groups actually consist of numerous individuals who are embedded in a network of fluctuating power relationships.

\section{Final Thoughts}

For a model to avoid representing practices as things, it needs to overcome three obstacles. First, it needs to solve what I have described as the problem of overlap. Rather 
than viewing a textual practice as an object, it needs to be imagined as a flexible entity with fuzzy boundaries. A satisfying model should allow for some ambiguity about how to define particular practices. Second, it needs to dissolve the issue of location, which is mainly a question of the verbs that we use to explain the learning process. Of course, practices are not like items that can be purchased at a store; as such, if we describe them as being "acquired" by students, we need to explain where they get these mystical practice-objects. Alternatively, if we imagine them as being "internalized," we are viewing them almost like molecules that are absorbed through a cell membrane; once again, the question naturally arises of where we might find these transcendent entities. Finally, the metaphor must somehow resolve the issue of change. Clearly, experienced writers are capable of utilizing their previously learned textual habits to accomplish tasks in new contexts. However, if we imagine practices as unified objects, it seems almost impossible to explain how a previously learned skill might be transformed by a writer to solve a novel problem.

While our discipline has a rich theoretical conversation about how to understand social context, many transfer studies have an unfortunate tendency to portray writing classrooms and academic disciplines as homogenous places with clearly defined boundaries. The most popular metaphor for understanding social groups is currently the notion of a discourse community, which is defined by a common "language" that is shared among members and gives meaning to their practices. While there is nothing necessarily problematic with this concept, it frequently overemphasizes what causes groups to cohere and remain stable. As a result, scholars sometimes neglect that social groups are heterogeneous. While it is possible to a certain extent to talk about a "shared" 
discourse, we also must realize that community members come from a variety of different backgrounds and are continually working to position themselves in more advantage ways. A group's boundaries are always open to renegotiation as people challenge existing social practices in order to meet the needs of new situations. Communities are therefore inherently historical entities that are inextricably caught in the flow of time. While moving beyond the metaphor of discourse community might be helpful in overcoming these difficulties, the important thing is to view groups as consisting of people embedded in a set of conflicting relationships.

Due to this tendency to commodify textual practices, the "transfer problem" is likely not as insurmountable as imagined by many researchers. Certainly, improvement can always be made to the existing curriculum, but caution is always necessary when making such sweeping reforms. In the next chapter, my focus will turn to the privilege granted to our conscious actions in transfer research. This tendency can be seen in our methodological approaches, which tend to problematically assume that self-reports about learning are accurate reflections of the actual process. However, in most current expertise research, scholars emphasize that conscious, rational decision-making is frequently the strategy most commonly utilized by novices; in contrast, experts tend to rely on widerange of intuitive habits that allow them to immediately assess the situation. My argument is that we need to acknowledge the importance of these unconscious behaviors. 


\section{CHAPTER III}

\section{THE MYTH OF THE CONSCIOUS WRITER}

The previous chapter examined the ways that transfer researchers represent practices as commodities, which students acquire over the course of their education and then sell to their employers. While scholars rarely explicitly advocate this position, it can be seen primarily in their use of metaphors-in many cases, practices are represented as physical things that learners "transfer" between different locations, almost like a truck shipping a product to a department store. The problem with this perspective is that it fails to capture several characteristics of social practices. In particular, it neglects the way that they often overlap and share resources—-while it might seem that writing a business memo and a short story are distinct activities, they both at least draw on certain basic syntactic skills, such as composing paragraphs and coherent sentences. Also, this model provides a limited sense of how learners change over time. For example, if practices are imagined as tools, then the only way for a student to learn is to acquire new ones. As a result, we frequently ignore how practices are transformed as students encounter new situations. Due to many of these theoretical preconceptions, researchers have come to see transfer as an intractable problem, mainly because they are unable to see how the "tools" acquired in a particular situation can be directly applied to the writing tasks in a different cultural context. 
One of the reasons that these metaphors are so prevalent is that they reflect a broader cultural ideology, one that also underpins how education is perceived by the general public, including the students that serve as the foundation for many of our studies. In much of the transfer research, participants continually demonstrate a tendency to conceptualize practices as discrete entities. In some cases, they seem to view writing as nothing more than memorizing rigid forms, such as the proper place to put a salutation on a letter. In other cases, they dismiss English courses as only teaching them to write fluffy, creative prose, which they view as being irrelevant to the factual writing in the disciplines. Much like the genre and transfer researchers, our participants also neglect to consider how practices are transformed as people move between contexts.

The purpose of this chapter is to demonstrate the importance of taking into consideration both conscious and unconscious cognitive processes when studying writing practices. Even if students believe that they learned nothing but "fluffy prose" from firstyear composition, they likely have developed several other practices without their conscious awareness. Therefore, if we limit our research to just retrospective accounts of their educational experiences, we risk producing a limited picture of what they learned in their writing courses. One way to overcome these difficulties is to use a wider range of methodologies. Composition researchers tend to privilege interviews, think-aloud protocols, and similar approaches, which predominately provide us with information about how our participants consciously describe their practices. We need to supplement this information with measures of the student's actual composing behaviors, such as student papers, revisions, classroom observations, and teacher comments. However, while broadening the types of data that we collect is a good place to start, the more 
important issue is that we need to reconsider how we interpret what students say about their experiences. When students fail to explicitly mention using a skill in particular situation, we should not necessarily take this as a sign that they failed to "transfer" skills from their previous courses - rather, they might no longer need to consciously attend to certain practices, so they might be focusing on different a aspect of their task. We need to situate student comments within a view of intelligence that takes into account the relationship between unconscious and conscious processes.

\section{The Duality of the Conscious-Unconscious}

While intuition might seem like a characteristic primarily of sports and other physical skills, most decision-making research suggests that it plays a central role in most of the professions. In Sources of Power: How People Make Decisions, Gary Klein discusses the results of studies of numerous individuals, and he concludes that the rational problem-solving strategies encouraged by many researchers are rarely practiced by people in many workplace environments. Instead of charting out the available options and logically assessing each possible choice, most professionals simply select what seems like the first suitable solution to a particular problem. As he explains, his initial assumption was that novices were the ones who "impulsively jumped at the first option they could think of, whereas experts carefully deliberated about the merits of difference courses of action" (21). He was surprised to discover that "it was the experts who could generate a single course of action, while novices needed to compare different approaches" (21). To provide a simple example, his research staff spent considerable time interviewing nurses to discover how they were able to determine whether babies contracted an infection. Astonishingly, many nurses can immediately discern whether a 
child needs to start an antibiotics regimen, even if tests from the hospital seem to indicate that he/she was healthy (40). When researchers asked the nurses how they could make such a rapid diagnosis, they were unable to explain the phenomenon; they responded that they were simply acting based on their instincts.

Much of our existing transfer research doesn't adequately take into account this tacit dimension of practice. The issue is not with a particular methodology—although we must recognize the limitations of certain approaches, the main difficulty is how we conceptualize the unconscious and conscious aspects of practice. "Unconscious" and "conscious" processes are not just separate trains moving along parallel tracks—rather, they are deeply intertwined, to the point that any analysis is incomplete if it fails to take into account their relationship. To use Etienne Wenger's terminology from Communities of Practice: Learning, Meaning, and Identity, the two aspects of the mind should be seen as forming a duality, which should not be confused with a dualism. The unconscious and conscious are not opposites, nor are they elements located on different ends of a spectrum, by which a behavior can be defined as more or less unconscious depending on the percentage of it that is conscious. As Wenger explains, the tacit is not "that which is not made explicit; the informal that which is not formalized; the unconscious that which is not conscious" (66). They are not behaviors locked in competition but ever-present aspects of the mind.

When we discuss the "unconscious," we typically are referring to a set of habitual processes that operate automatically without explicit awareness of the triggering cues (Bargh and Morsella 74). These unconscious processes work together to create an overall impression of our surrounding environment. Phenomenologists like to describe these 
fluctuating "impressions" as a person's lifeworld, which philosopher Evan Thompson defines as "the everyday world and the things that can be directly experienced within the everyday world—our living bodies, our natural surroundings, our cultural creations" (34). A lifeworld is different than brute physical reality, for it also includes all the psychological resonances in which we imbue the environment. To give a simple example, a door is nothing more than an arrangement of various molecules. But to a mind from a certain cultural background, the door is an object which can be opened to reveal other spaces. When we must confront a door, little deliberation is required to successfully use it; in fact, it seems to invite a particular action. The same thing typically holds with many linguistic behaviors. When we are caught in the flow of normal conversation, each word seems to automatically beckon the next one, causing the sentences to just emerge from the mind in a stream. While this process might be described as automatic, we should not confuse it with being unintentional. Just like a soccer player moving down the field, the speaker is guiding his words toward a fuzzy, undetermined goal. At times, these automatic processes will break down. At these points, our conscious awareness will redirect our attention, so we can modify our current behaviors.

However, this theory has no room for a particular conception of consciousness, which imagines it almost like a disembodied mind capable of objectively analyzing incoming sensory data. While we are capable of sliding their attention between different cues, we not capable of completely detaching ourselves from the flow of activity. In Cognition and Practice, Jean Lave demonstrates the numerous ways that the rational model of decision-making fails to account for actual problem-solving processes, even when people are engaged in seemingly mundane tasks like grocery shopping. As part of 
the Adult Math Project, her team of researchers observed twenty-five participants as they engaged in a typical trip to the supermarket. In particular, Lave highlights what she classifies as "best buy" problems, where shoppers need to make decision about which product is the best bargain. As she discovered, most people seemed to discern a solution upon discovering an appropriate frame for the problem. To give a simple example, one shopper discovered an unusually expensive package of cheese. From what he could infer from similar items, all the cheese packages were roughly the same size; however, the prices for the other packages were considerably lower, which made the high price somewhat unusual. He immediately surmised the price was a mistake. In order to verify this hypothesis, he searched for a cheese package of the same weight and compared their prices to search for a discrepancy (154). Rather than logically compare the prices through a mathematical formula, the shopper started with the solution and went through the process of making sure he was correct by using the available evidence. As Lave explains, "he was relatively certain which cheese package was inconsistent with the rest before he established whether there was really an inconsistency or not. The dialectical process in the particular context of everyday arithmetic is one of gap closing between resolution characteristics and information and procedural possibilities" (159). The shopper was not engaged in a linear process, where he initiated a procedure to discern the right decision. He instead recursively moved between the solution and the information provided by the situation, continually adjusting his problem-solving strategies to negotiate between these two poles.

In most of our daily practices, we only seem to initiate problem-solving processes when we hit a snag — when an aspect of the situation defies our expectations. As Lave 
points out, "repeated interactions produce a relatively smooth 'fit' between activity and setting, streamlining each in relation to the other, and generating expectations that the activity will unfold unproblematically and effortlessly. In relation to this expectations 'problems' take on meaning as conflicting possibilities for activity, or troubles with ongoing activity" (156). These "snags" bring what is an otherwise automatic process to our conscious awareness. Then, we are able to shift our attention to other procedures to resolve the difficulty. In the case of many writing situations, we might encounter such snags when we instinctively feel that a sentence is inadequate. Afterwards, we will start tinkering with it to resolve the difficulty. Alternatively, we might become stuck while writing a particular text, so we turn to models to see how other writers have responded to similar problems. Of course, we might also employ "rational" problem-solving practices, such as analyzing a text's audience, following certain rules, or constructing a linear outline - however, these constitute only one set of options, and they will not exhaust the possibilities.

If the "unconscious" is the set of habitual dispositions that work together to help us understand our environment, then "consciousness" seems to be how we direct attention to certain perceptual cues. When we hit a "snag" during an activity, we automatically begin trying to resolve the conflict, and we start initiating problem-solving procedures that we have developed throughout our previous experiences. In a similar fashion, we also seem to have the ability to redirect our consciousness attention, so we can deliberately focus on certain aspects of the environment that operate in the background. As Glen Mazis argues in Humans, Animals, Machines: Blurring Boundaries, one becomes "aware of the sense of what one is feeling .... but also is focused on the 
cause of those feelings and is able to 'move' in guiding one's attention here or there within experience" (158). Citing an example from psychologist Daniel Siegel, Mazis discusses a client who was feeling excessively agitated whenever she was feeling ignored in a conversation. After discovering that these feelings emerged from her neglectful mother, she was able to focus her attention to this aspect of her experience and understand how her irritation emerged from her troubled family history. We are similarly able to redirect our attention in more mundane daily activities. In the example of a soccer player, he might note a bothersome opponent moving in his peripheral vision, and he might choose to be especially wary of that particular person. In a classroom writing situation, we might have been told by a teacher to make sure that we perform a specific set of moves in our text, so we might make sure that we follow his/her directions. While we are always immersed in the flow of experience, we also have the ability to modify our behaviors through selectively choosing to focus on a specific aspect of the environment.

\section{The Trouble with Retrospective Accounts}

Without a vocabulary that highlights the interrelationship between conscious and unconscious processes, we risk overlooking certain factors when measuring transfer between contexts. In composition studies, the main problem is the lack of consideration of the tacit dimension of practice when analyzing our research data. Due to the reliance on think-aloud protocols and interviews, many studies focus exclusively on what is happening on the level of conscious awareness. In the case of think-aloud protocols, study participants usually discuss what they are explicitly considering during the composing process, which is often related to the most unfamiliar aspect of the current writing task. For that reason, they rarely discuss previously learned practices, mainly 
because such processes have been so well learned that they are deployed without any conscious reflection. Interestingly, when subjects make this argument themselves, researchers often dismiss the importance of their claims, even though students often mention that they no longer need to think about certain skills because they just "come naturally." In the case of research that employs predominately interviews, many researchers will neglect to see any "transfer" if the student is unable to remember specific details from previous course. Researchers take this as a sign they might not have learned the relevant skills. They also sometimes come close to conflating the participants' opinions about classes with their actual value.

When we ask participants to discuss their previous experiences, we must take into consideration that memories are not static objects that are simply brought into consciousness through reflection. Rather, they are actively constructed by the participant, who in the mere act of recalling an event can modify it according to the current circumstances (Siegel 42). For example, even though a student might never receive a single comment on mechanical issues in a writing course, he/she might recall his/her teacher as focusing exclusively on grammar because of his/her ideology about English courses. While such observations reveal a great deal about the perceptions of our discipline, we should not take them as direct evidence of what "transferred" from previous courses. Students probably are not fully aware of how their previous educational experiences affected their current practices. Much of their learning occurred on the tacit level and is not available for explicit recall.

To illustrate how such theoretical tendencies obscure what students have learned from their previous courses, let me begin by returning to a study mentioned in the 
previous chapter, Lucille Parkinson McCarthy's “A Stranger in Strange Lands: A College Student Writing across the Curriculum." While her methodology draws from a variety of approaches, she relies heavily on think-aloud protocols, which were administered when the student worked on the first drafts of his writing assignments. These were followed by thirty minute retrospective interviews where he was asked about his decisions. In the results, she found that the student focused on much different things as he moved between the courses. In freshmen composition, his main concern in the protocols was with textual coherence; in contrast, his poetry and science courses were more concerned with disciplinary content. Her interpretation of this phenomenon is suggested by her title. In her opinion, the data indicates he behaved like "a stranger in strange lands. In each new class [he] believed that the writing he was doing was totally unlike anything he had done before" (126). As she explains, he viewed the writing assignments in different courses "as being totally different from each other ... [his] characteristic approach across courses was to focus so fully on the particular new ways of thinking and writing ... that commonalities with previous writing were obscured" (137). Certainly, in both the protocols and interviews, the student often seems hopelessly lost when confronting a new rhetorical task and incapable of drawing on his previous experience.

The problem is that her analysis does not fully account for what might be happening outside of the student's conscious awareness. Even when he suggests that he might be instinctively drawing on previously learned skills, McCarthy's discussion largely ignores these claims. For example, when he struggles with his poetry analysis essay, his explicit focus is with producing a "correct" interpretation of the assigned poem. As such, the final draft mostly just summarizes the text, beginning with the first stanza 
and ending with the last one. When she asks him about whether he drew from his firstyear composition skills, he responds, "I didn't really have to think much about my thesis and subs because they just come naturally now ... But anyway it's not like in Comp like last year. Here my first paragraph is the introduction with the thesis, and the stanzas are the subpoints" (139). In her interpretation of this statement, she never addresses the fact that he claims to be unconsciously building on previous skills. Instead, she argues that "his focus on these new rules of use appeared to limit his ability to apply previously learned skills, the thesis-subpoint analytical structure, and kept him working at the more concrete summary level” (139). In fact, if the student never explicitly recalls using a skill, she seems to assume it had little impact on his work. As she explains, the student resembled "a beginning language user" (152). He might have learned to effectively write in certain courses, but these skills did not "automatically transfer to new context with differing problems and language and differing amounts of knowledge he controlled" (152). My contention is that even though he never explicitly mentioned previous writing practices, they likely functioned in the background, unconsciously operating as the student focused on what he needed to learn to complete each new task.

Because McCarthy doesn't account for the tacit dimension of social practice, her study also sometimes misinterprets the student's comments in his think-aloud protocols. Once we take these issues into consideration, her student no longer seems like a "beginning language user" who is continually leaving behind his old practices. Rather, he is simply allowing his existing practices to slip into the background while he focuses his conscious attention on the unfamiliar elements of the assignments. We should not be surprised that in the think-aloud protocols, his primary concern in his poetry class was 
"interpreting the poem and properly using quotes," while he concentrated more on "accurately understand[ing] the scientific terms and concepts" when analyzing a scientific journal article. We should anticipate this behavior from a student who hits "snags" in the writing process. Much like the supermarket shoppers who initiated certain strategies after stumbling into a discrepancy, the student was attempting to overcome the new challenges he encountered in his courses. His previously learned practices had not "vanished"; they simply were no longer the emphasis, for he needed to experiment with different writing strategies.

Furthermore, we should not be skeptical of "transfer" from previous contexts just because the student's abilities seemed to "break down" as he struggled with new tasks. In this student's particular situation, he had considerable difficulty with his poetry course, while he was much more successful with his biology assignments. However, this does not necessarily suggest that his practices sometimes failed to adequately "transfer" in his poetry courses. As Marilyn Cooper and Michael Holzman argued around the time of McCarthy's study, think-aloud protocols should not be viewed as accurate reflections of our composing practices. As they explain, "direct evidence of cognitive processes is unavailable.” Borrowing from one of Noam Chomsky's analogies, they argue studying writing practices is similar to "the impossibility of collecting direct evidence about the nature of thermonuclear reactions that take place in the interior of the stars" (284). At best, think-aloud protocols are demonstrations of what an individual was consciously attending to during the writing process. Without any additional evidence, we are unable to precisely determine the impact of the student's previous learning experiences aside from the fact that they made a contribution. 
While these issues are particularly evident in McCarthy's work, the tendency to dismiss the importance of the tacit dimension of practice is strikingly common among transfer researchers. For instance, in "Knowledge Transfer Across Disciplines: Tracking Rhetorical Strategies From a Technical Communication Classroom to an Engineering Classroom," Julie Dyke Ford examined whether students transferred skills between a technical communications and engineering course. In addition to conducting interviews and group think-aloud protocols, she also looked at papers and a reflective cover memo, where students discussed the strategies used to complete their final research report. The results indicated that while the students drew from the rhetorical knowledge from other courses, they mostly relied on "model-based" writing strategies that conceptualized genres as fixed forms. One of the students claimed that in their technical communications course, he learned "how a memo should look, how instructions should look, and how a report should look. So, [he] can get the spacing and the headers and all that stuff right, but [he's] still not sure how my words flow together" (309). In contrast, the students rarely mentioned abstract rhetorical concepts, such as audience, which many scholars consider essential to the writing process. As Ford observes, "Rather than let awareness of audience or sense of purpose be their guide to the other rhetorical strategies they relied on, the interviews suggested that the students instead seemed to first think about the concepts that were more tangible to them, model-based tactics" (310). Once again, the assumption is that writers should let considerations of audience consciously guide their actions; disapprovingly, she observes that "students did not reveal a tendency to plan and develop their own goals, nor did they show signs of considering alternatives" (310). The problem is that while the students might not have explicitly mentioned the notion of 
audience, a tacit feel for the engineering discipline likely operated in the background. In fact, one student directly claimed, "it was a given from the beginning of the semester that it was a technically oriented audience, so I didn't have to think a whole lot about it" (309).

One advantage of Ford's approach is that she collected a large sample of student texts, which can be used to determine whether her participants' interview comments are reflected in their finished products. They were evaluated by three doctoral students on a four-point scale, with a " 1 " considered "competent" and a "4" seen as "clearly incompetent" (307). Each text was examined by two readers, who rated its effectiveness in six areas: audience awareness, sense of purpose, organization, use of visuals, professional appearance, and style. Interestingly, despite the fact that the students rarely explicitly discussed audience and purpose in the interviews, the independent raters claimed they were competent in these categories. Overall, the students received a mean score of 2.08 in regard to their "sense of purpose," and only a slightly worse 2.10 in respect to their "audience awareness." If one were to evaluate the students' expertise based solely on these scores, they would seem to be mostly proficient writers. One of their least competent areas was organization, where they received a mean score of 2.56. Strikingly, this is the one area likely to be most impacted by their "model-based" composing strategies. If the students explicitly claimed to deploy any single organizational technique, it would be the process of determining the "parts" of a genre and using this formula to shape the text. As one student claimed, "I had to write a report like this in technical writing, so I'm going to look at how I set it up ... where the page numbers should go, stuff like that" (310). Alternatively, another student said that he 
would "go back and look at [his] technical writing book and see how [the report] should be set up, what format to use" (310). These observations offer some support for one of the key findings of contemporary expertise research—namely, that novices are the ones who most frequently rely on conscious deliberation, while experts follow their intuitive sense of what is needed in a given situation (Dreyfus and Dreyfus 30-35; Klein 21). The students seemed to have a "felt sense" of the appropriate audience, which is reflected by their high scores in this area. In contrast, their grasp of organization was on more shaky ground, which might explain why they turned to rule-driven strategies.

A study that avoids some of these difficulties is Laura Wilder's "'Get Uncomfortable with Uncertainty': A Study of the Conventional Values of Literary Analysis in an Undergraduate Literature Course." While her primary concern is not necessarily writing transfer, what is unique about her approach is her desire to understand the tacit practices at work in a particular classroom. She wanted to determine whether literary professors expect their students to employ the writing practices that previous researchers have noted are used by scholars in professional journals. To accomplish this goal, she looked at whether a class invoked certain special topoi, an Aristotelian term that denotes specific argumentative strategies used by a particular social group. She collected a wide range of data to determine whether class members employed these techniques. She observed class lectures and attended student conferences held by teaching assistants. To get a better sense of the rhetorical strategies used by students in their actual texts, she asked the TAs to select two successful essays, two "middle-of-the-road" ones, and two poor ones. Then, she had independent raters determine whether the papers were utilizing the special topoi. She also conducted interviews with the students that were identified as 
writing successful texts. Finally, she also distributed two questionnaires to all the students, one which was intended to look at what students perceived as their purpose when writing texts and another that attempted to determine whether students could identify whether certain passages engaged in literary analysis. Overall, she determined that the special topoi were frequently used in the classroom, both by the students in their written work and by the professor when he was explaining certain concepts.

The strength of her approach is particularly evident when she analyzes the professor's lectures, primarily because his explicit statements often clash significantly with his actual practices. At the beginning of the term, the teacher explicitly urges his students to avoid looking for deeper meaning in the texts, to the extent that he directly prohibits the use of many literary terms. At one point, he invites the class to analyze one of Frost's poems, and one of his students suggests that death is a prominent image. In response, the teacher points out that death is not even a word in the poem. When the student attempted to defend himself by picking out evidence to support his position, the professor interjected, "If we keep going, we'll underline every noun. We're calling the poet a liar; I know you said this, but you mean that" (186). He urges the students to "stay close, accuracy, think in terms of description, [rather] than interpretation" (186). Then, almost immediately afterwards, he engages in an extremely similar strategy. He informs the students that "now we'll make connections" and proceeds to interpret the poem's "pane of glass" metaphor as an Emersonian allusion. As Wilder points out, many of the students felt tricked by this approach, and they even felt he was discounting their literary expertise. 
At another point, when the teacher is discussing his assignments, he claims that his instructions were "purposefully vague" because he wanted to encourage creativity among his students. Nevertheless, he seemed to be looking for them to deploy some specific rhetorical strategies. After they turn in their first assignment, he chides them for "symbol hunting" rather than engaging in the complex kinds of interpretation that he has been encouraging throughout the course. He even pushes students toward directly using certain special topoi. At one point, he urges them to look for paradoxes, claiming that "what one writer finds corrupt may be ideal in another work [is] the best kind of argument ... take [the] questions and make [them] more specific, more complex" (194). In fact, throughout the course, he seems to have an overriding concern for complexity; he's continually demanding that students deepen their analysis of the texts. Obviously, if Wilder had just directly interviewed the instructor, she would have only gained a limited picture of his literacy practices. To get a better sense of what was happening on the tacit level, she needed to examine his behaviors and compare them to his explicit observations.

To give another example of how triangulating between interviews and other data richens our understanding of writing practices, Wilder frequently compares the students' interview comments to the revisions that they made to their texts. Interestingly, she discovered that while students frequently believe their writing changed little throughout the term, they would slightly adjust their practices in response to the feedback that they received on their early drafts. In one of the student's essays, he argued that a poet was using "sarcasm" to convey a message. The TA strongly discouraged the use of this term. Instead of taking this approach, he highlighted a moment where the student employed the word "mask": "What you were arguing more effectively was that Bradstreet adopts a 
mask of humility in order to publish her poem but we realize through her words that it's only a mask to cover her frustration" (204). Even though the student had only occasionally used the word "mask," he proceeded to use it extensively in the final draft, even though he admitted in the interview that he never felt this changed his overall argument. At another point, a TA felt that one of the student's papers was overly simplistic, a judgment that was verified by the low complexity score given by the independent raters. In the final draft, the student mostly retained his original argument. Interestingly, though, he deleted all statements that suggested a simplistic interpretation, including phrases like "it's perfectly clear." While this is not an enormous revision, it indicates that the student understood that "complexity" was valued by his instructors. Wilder continually documents moments where students make subtle behavioral shifts, which often occurred without the students' conscious awareness. I would argue that such little changes - employing the use of a different word, cutting out particular phrases - are what produce larger transformations. If Wilder had not deployed a methodological approach that was sensitive to these shifts, she might not have ever detected these small variations in their practices.

Wilder's study highlights the central difficulty in educational research-namely, the fact that practices change slowly over time, and learners are often not consciously aware of these transformations. If most of us were asked when we learned to write a coherent sentence, we would not be able to provide a specific answer. Most likely, we would talk in a vague way about what we were taught in elementary school. While some of us might be able to recall specific memories, most of our observations would just be reflections of the dominant way of thinking about teaching writing to young children. To 
use an analogy, asking someone to pinpoint when they learned certain practices is similar to getting them to reflect on changes in their personal appearance. If a person has gained weight over the course of several years, they might have a general sense of how they got to that point. They perhaps could indicate that they "ate too much," but they would not remember the precise meals, aside perhaps from particularly memorable moments during vacations and special events. They also might not be aware of other contributing factors, such as the life stress that comes from moving to a different location, raising young children, or starting a new job. In fact, we are rarely aware of changes to our bodies until someone points it out. In the same fashion, we are continually adjusting our practices to meet the needs of new situations, often without the recognition that these slight modifications are part of a larger transition. To design a study capable of detecting such gradual shifts, we must do more than just conduct interviews or think-aloud protocols. We also need to observe actual student behaviors, without which we risk missing details that elude the people immersed in a particular practice.

\section{The Interpretation of Retrospective Accounts}

While acknowledging the importance of the unconscious dimension of practice is a step in the right direction, the main challenge is developing a more adequate model of how students' explicit statements about the composing process influence their tacit practices. Some connection obviously exists between these two dimensions. If a person thinks "good" writing should be concise, this presumably should cause him/her to be more diligent about cutting unnecessary words. But the relationship between the two is not simple. From the existing transfer research, we know that students tend to have a particular view of the writing process, one that acknowledges the importance of revising 
before submitting a final draft. On the other hand, we also know that students often fail to follow this advice; many choose to complete the text the night before the due date, which allows little time for revision (Bergmann and Zepernick 137). For many students, the traditional model of the "writing process" operates like a set of moral commandments: students believe they should produce multiple drafts, even if they continually violate this principle. The point is that it is not entirely predictable how abstract beliefs influence actual behaviors. When a student claims that he/she learned to "always revise before they turn in an essay," this is not evidence that certain practices "transferred" from his/her previous writing courses. At best, we can argue that the student learned a certain ideology, one likely taught in high school and then reinforced in college courses. To understand how students' practices are related to these explicit claims, they must be viewed in the context of the other elements of their life, such as their disciplinary interests, cultural background, and previous educational experiences.

These problems were originally noted by critics of early studies that draw heavily on interview data. In "Talking about the Composing Process: The Limitations of Retrospective Accounts," Barbara Tomlinson argued that we are particularly accepting of student reflections about their writing process. When we look at interviews of published authors, we take for granted that they are engaged in a performance, one that often reinforces our tacit beliefs about creativity. We are much less suspicious when we turn our gaze to writers who are not professionals. We tend to neglect the ways they are also engaged in a performance, one that reflects certain aspects of their life and how they situate themselves in the university environment. Citing an assortment of psychological research on retrospective accounts, Tomlinson points out that participants sometimes 
report on cognitive processes "not by referring to memory, but to the most available 'a priori theories' about what those processes might have been" (440). The issue is not that memories are "distorted" by other considerations. Rather, whenever someone recalls an event, it is always interpreted in light of his/her present circumstances. Therefore, what we learn from retrospective account is not how student actually completed a task but "their conceptions of how one should think and talk about writing processes" (442). Looking back at the results of Ford's study, what is interesting is the prevalence of certain ideas about writing, some of which seem quite dismissive of the entire practice. One student directly stated, "What is the point of taking technical writing when all you are going to do is use templates afterwards?" (309). Many students sharply distinguished between "writing" and "engineering" tasks, to the point of claiming that "in my technical writing class we had to do an article review, but that experience won't help me any here. This assignment is a lot more concept-oriented" (306). One of the main reasons that students continually mentioned "model-based" strategies is because they viewed writing as dealing entirely with form. They mostly seemed to feel that writing was a matter of mastering inane trivialities, such as the correct format for "the spacing and the headers" and the necessity of initializing business memos (309). While these accounts provide valuable information about how writing is perceived among engineering students, their actual practices certainly extend beyond these limited confines.

Unfortunately, most transfer research rarely considers how such accounts are influenced by cultural factors, such as peer groups, disciplinary ideologies, and previous educational experiences. In "Disciplinarity and Transfer: Students' Perceptions of Learning to Write," Linda Bergmann and Janet Zepernick discuss several focus groups 
conducted with upper-division undergraduates asking about their perceptions of first-year composition. Their primary mission was to discover what skills "transferred out" from the students' previous courses. The results indicated that students perceived composition classes as involving expressive writing, even though some the students likely took courses with a focus on argumentation. Such writing was viewed as flowery and excessively focused on correctness. In contrast, writing in disciplinary courses was perceived as fact-oriented and less concerned with mechanics. While Bergmann and Zepernick acknowledge this study merely measures the students' perceptions of the firstyear course, both the questions in their focus groups and their own analysis neglects to consider other cultural factors that might have produced these interview responses, mainly because they are not perceiving writing development as interacting with the other elements of the students' lives. To offer one possible explanation for their data, it was collected at a technical college, where the majority of these students were engineering and science majors. Much like in Ford's study, the students might have developed a low opinion of what happens in English studies, which is often seen as intellectually suspect in comparison to the rigorous hard sciences. While the students claimed their composition course involved mostly mushy expressive writing, their comments might just stem from being acculturated into a discourse that does not value work in the humanities. I would argue that what is remarkable about Bergmann and Zepernick's study is not the lack of transfer from first-year composition; without utilizing a methodology that examines how students actually go about completing certain tasks, we have no way of knowing how they draw from the tacit practices they have acquired throughout their 
lives. The more interesting finding is how little respect literary studies and composition is granted by students from other disciplines.

One of the reasons that Bergmann and Zepernick never consider this possibility is because of the privilege they grant to student comments. In fact, they explicitly open with the premise that they "repeatedly observed a tendency among student to actively reject the idea that what they learned about writing in high school or first-year composition (FYC) course could be applied to the writing they were asked to do in the disciplines" (124). Rather than being skeptical of such claims, they chose to "take these comments from faculty and students seriously, as indicating that students may not be transferring to their upper-level writing experiences the knowledge that we hoped they had acquired in the earlier stages of the process" (125). However, they never fully explain why we should take these student observations as evidence of what practices actually "transferred" from their writing courses. Later, they even acknowledge that the students' stories should not be taken as "accurate representations of their actual process of literacy acquisition" (126). They argue that they should be viewed as 'representations of students' own perceptions of how and where they learned to write and, most of all, what students believe themselves to be learning — what knowledge and skills they understood themselves to have acquired as thinkers and writers" (126). Despite this confession of their study's limitations, they only briefly consider how a student's other practices might encourage a particular viewpoint on writing courses. At one point, they suggest that the students' beliefs might be attributed to the culture of the engineering discipline, "which tend[s] to have a tradition of 'toughness,' passed along to both faculty and peers" (138). Nevertheless, 
Bergmann and Zepernick never fully investigate this possibility, partially because they never inquired into such issues in their interviews.

A similar problem can be seen in Anne Beaufort's College Writing and Beyond: A New Framework for University Writing Instruction - except, in this case, she is frequently critical of the student in her study, but only when he raises questions about the pedagogy practiced by his disciplinary instructors. As I mentioned the previous chapter, the study follows the work of a white male student as he moves between his first-year composition, history, and engineering courses. Over the course of her research, she collected a massive amount of data, including 20 hours of interviews, 30 hours of classroom observations, and 100 pages of student text. When she examines the student's work in his first-year composition course, she is extremely dismissive of the classroom pedagogy — to the extent that when the student's observations conflict with the teacher's written feedback, she often assumes that he is the one with the correct interpretation. In respect to an assignment where the student wrote a letter to a film director, she notes that the teacher praised his work and wanted to include it in her classroom reader. In contrast, the student described it as "some dumb thing," which Beaufort interprets as indicating that "it was not a piece of writing he took seriously in terms of his stated audience" (37).When her attention shifts to his history courses, she is less prone to make such judgments, even when the student's comments are similar to those he made about his writing course. She usually defers to the expertise of his disciplinary instructors. In the interviews, the student described his work in an Islam course as "an exercise in regurgitation. What we could tell we had learned or know" (67). Upon reviewing the TA's comments on one of his papers, the student claimed that "in order to get the grade 
on the paper ... you had to say what you'd been told about the book. Maybe in a new way, maybe in more depth, but basically say the same thing" (67). These observations could be interpreted as providing more support for the idea that his writing was about "doing school." Instead of reaching this conclusion, Beaufort interprets the student as making baseless complaints. Upon examining the student's actual essays, she observes that they demonstrate serious organizational problems, such as the lack of a focused thesis statement. She even blames these issues on epistemological differences with his first-year course, where "truth was something to quest after but with an open mind, looking at many possibilities" (64). In contrast, his hard-nosed history professors sought "greater certitude ... to be credible, in the history classes Tim was taking, it appeared one needed to make a clear-cut argument" (64). Of course, she never questions whether encouraging this practice is desirable for a history student, nor does she look at the student's letter to the film director to see if it was "some dumb thing." She seems more interested in illustrating the pedagogical problems with the approach taken in his writing course.

When we neglect to consider how various ideologies might influence the comments of our research participants, we risk inadvertently reinforcing the values of certain cultural practices. Because Bergmann and Zepernick's study was conducted at an engineering institution, the students seem to believe the English discipline has no practical value. If we adjust our practices to these students' views, we are encouraging the values that underpin this discourse. Many seem to have embraced the idea that the skills students learn in their university courses should somehow immediately translate into capital that can be earned in the workplace. They imagine their writing practices as 
transferable commodities that can be directly sold to employers. Because this ideology is hidden behind the veneer of empirical research, we are less likely to question it; we fail to interpret the students' comments in light of the broader cultural landscape. Then, when we start to think about how to reform the curriculum, we are continually asking how we can conform to their expectations, even when their views of writing are problematic. We need to acknowledge that various ideologies shape the comments of our participants. While it is important to have skepticism about our own pedagogical practices, it is equally important to not just accept our participants' views without fully considering their implications.

The issue is that a practice is not capable of being fully captured by a linguistic representation. When participants describe their behavior to a researcher, they must translate their phenomenological experience into explicit terms. These descriptions will be shot through with various cultural ideologies, which provide hints as to how participants position themselves in relationship to other social practices. In most cases, these descriptions will also serve a pedagogical function — much like teachers attempt to find a way to describe unfamiliar practices to students, interview participants must try to explain their perspective to outsiders with different experiences. While such information is certainly useful, it does not necessarily provide direct evidence of a person's conflicting cognitive dispositions. As Bourdieu points out in Outline of a Theory of Practice:

The relationship between informant and anthropologist is somewhat analogous to a pedagogical relationship, in which the master must bring to the state of explicitness, for the purpose of transmission, the unconscious 
schemes of his practice. Just as the teaching of tennis, the violin, chess, dancing, or boxing breaks down into individual positions, steps, or moves, practices which integrate all these artificially isolated elementary units of behaviour into the unity of an organized activity, so the informant's discourse, in which he strives to give himself the appearances of symbolic mastery of his practices, tends to draw attention to the most remarkable "moves"; i.e., those most esteemed or apprehended. (18-19)

When participants reflect on their actions, they are being asked to discuss them in a highly artificial research situation; as such, these descriptions can only provide indirect evidence of the nature of their practices. They might be revealing which behaviors are most respected by people in the larger community. Their comments might reflect how they differentiate themselves from other cultural groups. They might also indicate their immediate preoccupations, which will provide some insight into how they are consciously orienting their actions. We need to take all these factors into consideration when we are analyzing retrospective accounts of writing practices.

The point here is not to discount the value of interview-based methodologies. Rather, the goal is to situate them within a more inclusive understanding of the components of human intelligence. If we ask participants to explain their writing decisions after completing a particular assignment, we are likely to hear primarily their post-hoc rationalizations of their writing process. Due to the value our culture places on conscious deliberation, they might be inclined to describe their choices as that of a rational actor who logically chose between competing alternatives. Alternatively, some participants might have learned to imagine writing as a romantic process, which might 
cause them to emphasize their moments of spontaneous, creative inspiration. While neither of these descriptions provides a complete account of their actual linguistic practices, they do provide some insight into the explicit ideologies held by many of our students. To put this another way, these approaches are similar to reducing an animal's intelligence to its observable movements. Underneath the skin, an entire system of muscle, bone, and tissue work together to help it make decisions based on specific environmental cues. In the same fashion, we should avoid reducing writing to its most easily measurable surface elements. Instead, we need a framework that takes into account the rich tapestry of intellectual dispositions that operate in the background to produce what we recognize as a competent linguistic performance.

\section{Final Thoughts}

The main problem with the existing studies is the assumption that verbal accounts provide a direct window into tacit cognitive processes. Even when data is gathered from multiple sources, the tendency is to not even consider evidence that conflicts with the students' explicit reports. However, once we look closer at these studies, we have numerous reasons to believe that the information provided by students is not an accurate description of what they learned from their courses. Many of their observations about first-year composition suggest the widespread use of discredited pedagogical practices, such as a focus on mechanical correctness and generic textual patterns. While it is certainly true that some teachers focus exclusively on these concerns, it is unlikely that such attitudes are as prevalent as indicated by the students. In addition, many respondents also express that first-year composition deals mainly with expressive writing, which often puzzles researchers who are intimately familiar with the curriculum at the research site; 
these claims arise even in programs that are explicitly focused on academic argumentation. Finally, the students' responses reveal evidence of many stereotypical beliefs about the English discipline. Rather than view it as involving serious intellectual work, many students consider it a field that develops obtuse rationales for what are largely subjective aesthetic opinions. Just as teachers have no right to interfere with a "personal" reading of a literary work, they should not expect a student to change their "individual" writing style. Judgments about such issues are seen as a matter of taste. Interestingly, while we are generally critical of such statements when made by people from outside our field, we are less prone to question such opinions when expressed by students.

We need to be especially cautious when students discuss the struggles they experience in their courses. As I pointed out in the last chapter, the tendency among transfer researchers has been to interpret any sign of conflict as an example of "negative transfer." If we view practices as commodities, this perspective makes sense- the main reason a student would fail to learn a practice is because something has obstructed the delivery, such as when a clog in internet bandwidth prevents someone from downloading a file. The emphasis in transfer research on making connections between situations causes any difference to be interpreted as a potential obstacle. Looking at the issue from the perspective of contemporary practice theory, we see that student are not acquiring a skill —instead, their practices are in constant development as they respond to other practitioners. The next chapter outlines a model of practice that takes into account such considerations. 


\section{CHAPTER IV}

\section{THE RHETORIC OF ALIGNMENT}

In "'Mutt Genres' and the Goal of FYC: Can We Help Students Write in the Genres of the University?," Elizabeth Wardle recounts the experience of a second-year MA student teaching a learning community section of first-year composition that was directly linked to a biology course. Instead of just using the assignments typical of introductory writing courses, she wanted to design a curriculum that directly prepared students for writing in the biology discipline. Most of her fellow teachers required student to write summaries of research articles, but she thought it would be more useful for students to produce abstracts due to their ubiquity in the hard sciences. She was also skeptical about the value of teaching students to write standard argumentative essays. She felt it was more important for students to learn how to construct "biological arguments." Given that she was unfamiliar with biological discourse, she began the process of building such a curriculum by consulting with her colleague in the attached biology course. As she explained, "I want to learn more about what knowledge is valued in [the biology professor's] field and how writing in the field of biology is supposed to be, what their genre is like, because that's what I would like to teach students" (779). Once she got "all this discipline specific knowledge," her goal was to "meld as much of into [her] class as possible" in the hopes that students would be better prepared by directly engaging in the writing practices of biological scholars. 
I want to describe this line of argumentation as the rhetoric of alignment. While composition scholars rarely exhibit this reasoning in such an explicit form, it underpins many of our debates, from the argument over explicit teaching to the proposals for reforming first-year composition. This model likely originated in the industrial economy, where workers must continually repeat the same tasks. Given that an industrial worker rarely needs to adapt to new situations, job training mostly involves preparing novices to engage in a narrow range of codified behaviors (Kalantzis and Cope 126). The goal therefore becomes aligning the curriculum as much as possible with what is expected in the target context. Applying this approach to writing instruction, the idea is that we should require students to engage in tasks similar to what is expected in future contexts. If we anticipate that students will write resumes, they should be given opportunities to practice in this genre.

The problem is that this model of education only describes an extremely limited range of practices. While our lives certainly involve continuously repeating a fair share of codified behaviors, the truth is that the contemporary workplace frequently requires us to adjust to new contexts. Unfortunately, many of our metaphors for learning remain mired in this industrial mindset. As I argued in the first chapter, many genre scholars and transfer researchers have a tendency to use metaphors that represent practices and contexts as commodities, as unified things that students purchase in the educational marketplace and then trade to employers for economic capital. When we view a practice like a commodity, we see it as an object with a set of well-defined characteristics. The act of teaching then becomes explaining these features to our students. Alternatively, when we view a context as a commodity, we see it as a location with a fixed set of boundaries. 
Our goal then becomes helping students understand how to operate within these confines. While this sort of reasoning is well-suited for preparing workers for industrial work, the problem is that the situations that students face in the university are not such activities. When a history student is asked to write in his/her courses, he/she does not necessarily see each professor as engaged in a "discourse" with a shared language for talking about the world. Rather, he/she sees an assortment of idiosyncratic professors, each with their own set of expectations, which he/she must learn how to negotiate with to successfully complete his/her degree. The truth is that most professional writing practices operate in this fashion. They are not codified, repetitive behaviors but rather flexible strategies that are built to respond to a variety of situations.

Returning to the example in the opening paragraph, the problem with the instructor's approach is not necessarily that she lacked the experience to adequately teach students biological discourse. Of course, her class was certainly divorced from the act of conducting scientific research. As Wardle points out, the students "could not write [biological] arguments because the arguments depended entirely on research the students were not able to do the first-year of in college - because they were not involved with the activities of biology" (780). As the teacher explained, "they hadn't done the kind of thing that would merit writing in that way. What they had done was researched about biological topics, mostly about genetically modified somethings" (780). But the challenges run deeper than even this issue. Even if the teacher were capable of having students conduct some research, she still could not adequately teach them to write like a biologist. This discourse is not just a thing that can be handed to students. Rather, what we describe as "biological writing" is the product of a collection of frequently conflicting 
dispositions. The student learns this practice by actually interacting with specific people in the world. Rather than acquiring a "stabilized-for-now" genre, the student discovers that the discourse is not stabilized and that his/her writing practices must continually accommodate new situations.

Looking at the issue from this perspective, the rhetoric of alignment is somewhat unproductive. When we are attempting to direct the curriculum toward a well-defined objective, this way of thinking makes some sense-like a construction worker building tracks to a specific destination, we can ultimately orient all our classes toward moving students to a single unified point. However, once we begin to see a practice as not being stabilized, this rhetoric starts to become problematic, for the destination is not a clearly marked location but an ever-shifting target. The student is not just learning to do one kind of biology. Rather, they are discovering several ways of doing biology as they move between courses and become acquainted with new teachers. Becoming a proficient biological writer means actually being acquainted with these differences. Furthermore, steering students entirely into biological courses might also be problematic-after all, understanding biological writing is not just about encountering the narrow range of practices taught in biology courses. The student must also understand how biology differs from other practices, such as those prevalent in the humanities disciplines. Learning to write involves discovering how practices are embedded in a network of conflicting relationships.

This chapter attempts to move beyond the view of practices as being stabilized, unified entities. Rather, I argue that we need to look at practices as collections of loosely related dispositions that are deployed through interactions with specific people in the 
surrounding environment. In the first section, I take a closer look at what I describe as the doctrine of sameness, showing the ways that transfer theorists have privileged recognitions of similarity in the learning process. In the next section, I claim that we need to move toward a model of practice that views it as a temporal "coupling" between an individual's dispositions and his/her social environment. I focus particularly on the importance of how people interpret their social world and sell their ideas to other people. In the final section, I argue that social actors are best imagined as virtuous that are continually in the process of modifying their practices to meet new demands.

\section{The Doctrine of Sameness}

The rhetoric of alignment encourages us to view learning as primarily consisting of repeating behaviors across similar situations. In "Rocky Roads to Transfer: Rethinking Mechanisms of a Neglected Phenomenon," Gavriel Salomon and David N. Perkins offer a typical formulation of this argument. As they explain, an adequate definition of transfer must begin by distinguishing it from "mere" learning, which they define as an action repeated in almost identical contexts: "When learning something leads to a later performance we identify as more or less the same in a context we identify as more or less the same, we do not call this transfer, we just call it learning" (115). As an example, they utilize students memorizing dates for an exam. Their test performance would not be considered "transfer," even though some minor differences exist with the memorization situation, such as the fact studying usually doesn't take place at school. By describing the process as "mere" learning, they convey the impression that it is ubiquitous — in their view, most learning occurs through this process. Given that is the case, any difference between the classroom and future contexts is a potential obstacle to a student's education. 
Operating under this principle, many composition scholars have advocated using "real world" assignments, with the idea that students will learn more effectively if teachers minimize the difference between the classroom and professional environments. The problem is that two situations are never identical, which means that we can always specify an innumerable number of differences. To give just one example, classroom writing is always evaluated by the teacher, while workplaces have more diverse audiences. In addition, while the goal of many classrooms is the student's learning, many workplaces focus on achieving practical material objectives. As Joseph Petraglia has argued, even when students are asked to respond to "real world" situations, they are at best only "appearing to address an audience, looking like [they] have a purpose, and pretending to be knowledgeable" (92). In the case of first-year composition, scholars observe that disciplinary classrooms involve highly specialized discourses, which are usually outside the knowledge of most writing instructors. As Wardle points out, "everything we have learned as a field over the past decade suggests that specialized writing is best taught by reflective insiders who know the genres and their content" (783). While researchers mention other discrepancies, their main argument is that transfer is difficult because of the lack of alignment with future contexts.

Similar assumptions underpin Wardle's study of the prevalence of "mutt genres" in first-year composition. Her data was collected from "learning community" writing courses, which were linked with other classes in the students' major. She conducted focus groups, surveys, and interviews with both the students and the teachers; in addition, she examined their first and last papers along with other written materials, such as a rhetorical analysis and a cover letter where they discussed the assignment's objectives. 
Finally, she looked at the assignment sheets from the courses. While the university offered some broad guidelines, most of the curricular decisions remained in the hands of the instructors. Despite this considerable amount of freedom, she discovered that the teachers offered extremely similar assignments. She notes the prevalence of many common genres, including personal narratives, profiles, interviews, travel narratives, evaluations, reflections, and rhetorical analyses. In particular, she chooses to focus on observation and argumentative essays, which she believes indicates some of the broader problems that exist in many first-year composition courses. While these assignments are intended as "practice" for future writing tasks, they often only share superficial similarities with the work done in other disciplines. She describes such assignments as "mutt genres," which she defines as "genres that do not respond to rhetorical situations requiring communication in order to accomplish a purpose that is meaningful to the author" (777). For instance, the official description of the observation essays insists that "paying attention to specific details is the basis of good descriptive writing" (774). Even though "observation" is required in many disciplines, it often denotes very different skills; in the natural sciences, "observation" means abiding by certain experimental methodologies, while it might mean attending closely to the text in literature courses. Once again, she considers these differences to be obstacles to the student's education.

The problem is that we can always specify numerous differences and similarities between any two contexts. According to Wardle, first-year students are in a unique rhetorical situation, one that involves producing a text entirely for a teacher-evaluator who will somehow rank their discursive performance. Other tasks presumably require more complex audiences and serve more practical purposes. While some truth certainly 
exists to this claim, it neglects to consider how contexts are linked through numerous similarities. When students move into future courses, they will continue to produce texts for other teacher-evaluators. After graduation, most will move into businesses with bossevaluators, who might not utilize the same criteria but who will nonetheless be judging their performance. Even if our students attain a management position, their work will always be subjected to this ranking impulse, even if it involves little more than comparing different incomes. As Bourdieu argues, social institutions are intimately linked together through a set of homological relationships and therefore tend to reinforce similar dispositions (Invitation 105-06). At the same time, social groups consist of members who are continually shifting their allegiances and moving between institutions, so could also specify numerous differences between any two contexts. Any sense of how "contexts" operate must take into account the temporal nature of experience. A "context" must necessarily refer not to a stable structure but rather to a network of social relationships that is continually transformed as it moves through time.

Just as Wardle portrays contexts as unified structures, the mutt metaphor suggests a similar understanding of writing practices. The term is most commonly applied to animals, where it describes a combination of two different "pure" breeds. While the idea of "pureness" might be useful when selling animals in the marketplace, no animal could be characterized as being "pure" genes from a historical perspective. Throughout a breed's history, it has been continually transformed through its interaction with other animals; its genes have always been "polluted" by other breeds. Genres similarly never exist in a "pure" form. When we are talking about a "genre," we are actually referring to a loose label for a set of related writing practices. We could always point out an almost 
innumerable number of differences between two writing practices, even if they involve people writing within the "same" genre. Due to the fact the world is in flux, practices will always be somewhat different. One of the difficulties of transfer research is that some arbitrariness will accompany any attempt to devise a metric for the sameness of two contexts.

While some work has been done to resolve this issue, little has been accomplished beyond the development of more flexible measures. For example, Salomon and Perkins recommend looking at the distance between two situations. In one example, they describe a situation where someone has learned how to drive a car and then finds themselves behind a truck. We might classify this situation as "near" transfer because of the numerous similarities. At most, the driver may have to adjust to the fact that the vehicle requires more space to make certain maneuvers. As Salomon and Perkins emphasize, the methods for teaching in such instances are relatively well understood, and many educators intuitively utilize these practices in the classroom. Most of them involve aligning the curriculum with what is expected in the target context. The more difficult question is how to facilitate transfer between distant situations, such as going from driving a car to flying an airplane. They suggest "bridging" activities, which help students derive abstract principles that can be imported into new contexts - as an example, they describe a teacher discussing the similarities between home plumbing and the human circulatory system (Perkins and Salomon, "Science" 7-8). Regardless, the larger problem is adequately defining "distance.” To a certain degree, transfer researchers have recognized this difficulty, but how they deal with it merely highlights the limitations of the traditional framework. In a moment of puzzlement, Salomon and Perkins confess that 
their definition of transfer contains a "suspicious circularity" ("Rocky Roads" 115). Referring back to their distinction between transfer and "mere" learning, they point out that no obvious metric exists to help us make a distinction between these processes; after all, how people determine the distance between situations often depends on their subjective opinions. Nevertheless, they neglect to consider what challenges such difficulties pose for the concept, choosing instead to acknowledge that transfer is more likely to be mentioned when something mysterious happens during the learning process that was not anticipated by researchers.

Interestingly, claims of "unpredictability" are surprisingly common, particularly in discussions of transfer between different situations. While David Smit acknowledges that transfer is a regular occurrence, he claims that the problem is that people "do so at all levels of abstraction, and we cannot say much about this phenomenon except that it indeed occurs. That is, we know little about the mental processes involved and can generalize very little from what we can observe" (132). In much the same fashion, cognitive scientist Douglas Detterman goes so far to argue that "novel insights as cases of transfer are probably rarer than volcanic eruptions and large earthquakes. Like any other rare event, important cases of transfer are difficult to study because no one knows exactly when or where they will occur" (2). He relegates true acts of transfer as the product of occasional genius; in his mind, "very little human behavior is novel and of great significance to the future of humanity" (4). Of course, this argument does little to clarify how students approach "distant" situations. The hypothesis of many transfer theorists is that people cope with new challenges by transferring skills learned in previous circumstances. If the end result of this approach is that such transfer involves a 
mysterious process, then we have substituted a scientific explanation for some kind of magic. The solution is not to abandon hope but rather to revise our framework.

\section{A Model of Practice}

Time is the main factor that confounds any attempt to develop an adequate metric of sameness. Once we take into account that the world is always in motion, it becomes impossible to determine in a rigorous way whether two situations are "similar enough" to encourage transfer. As I mention in the introduction, Bourdieu has been criticized by many theorists for being deterministic, but one advantage of his approach is that temporality is placed at the heart of his model. Following the work of Omar Lizardo, I see Bourdieu as stipulating a duality of structures. He views the habitus as a cognitive structure, a conflicting set of dispositions that shifted in response to cues from the environment. The habitus is in a dynamic relationship with the field, which he views as a group of individuals embedded in a shifting set of power relationships. To better understand this model, it might help to draw an analogy with what Salomon and Perkins use as the paradigm example of a routine practice, which is driving a vehicle. If we view driving through the lens of traditional transfer theory, we would argue that it is routine because people are always repeating the same behaviors in similar situations. This way of thinking captures a certain truth—when we step into a car, we always find the steering wheel in the same place, and we always push on the accelerator to achieve momentum. But the striking fact is the enormous number of differences that exist between driving situations. As we move down the street, we encounter cars embedded in a set of shifting relationships, and we must adjust our behaviors to their movements. On a regular basis, we encounter entirely novel situations, such as when a person suddenly dashes across the 
street without waiting for the walk signal. Nevertheless, we are usually able to adapt to these situations, for a practice provides more than just a set of habits — as Bourdieu observed, it also allows us to improvise. If we use the driving example to understand Bourdieu's framework, we might describe a driver as the habitus, the individual's dispositions that allow him/her to adjust to the actions of other people. The other vehicles might be characterized as the field, a group of people embedded in a set of changing relationships.

Transfer theory helps enrich Bourdieu's approach by bringing greater clarity to the learning process. To address some of the difficulties in traditional transfer studies, recent researchers have introduced actor-oriented theories, which argue that we must begin by looking at how learners construct similarities between situations. While this approach takes into consideration how the student changes over time, the problem is that it frequently fails to take into account his/her relationship to the social environment. In Joanne Lobato's formulation of this argument, transfer is "the personal creation of relations of similarity, or how 'actors' see situations as similar" (18). Slightly complicating this idea, Ference Marton has argued that we must also consider how learners perceive differences. These theories improve on the traditional framework by acknowledging that similarities are individual constructions and not independent properties of the world. Along the same lines, composition scholars have invoked a variety of other metaphors that stress the importance of recognizing similarities. Drawing from the work of activity theorists, Wardle has suggested that we should move away from the notion of transfer and toward the idea of generalization. She argues that the concept goes beyond just "carrying and applying knowledge across tasks" to include how 
individuals "construct associations among social organizations, associations that can be continuous and constant or distinctive and contradictory" (68). This metaphor is more powerful than the idea of just similarity, for a generalization requires the recognition of both similarity and difference — when learners make a generalization, they are recognizing a continuity between distinct situations, like when a person notes that the wind blows before it rains. She also emphasizes that the student exists in a relationship with his/her social environment, although her focus still seems to remain on the individual.

In Agents of Integration: Understanding Transfer as a Rhetorical Act, Rebecca Nowacek offers a more complex version of the actor-oriented theories, arguing that we need to take into consideration not just the connections made by students but also how they are received by other people. To support this claim, she examined a three-semester sequence of interdisciplinary courses at a Catholic university, collecting data ranging from class observations, interviews with professors and students, videotapes of class periods, and copies of student essays. She discovered that although students were continually making connections, they were not always recognized as legitimate by instructors. As she explains, students must "learn not only to 'see' connections among previously disparate contexts but also to 'sell' those connections, to render them appropriate and convincing to their various audiences" (39). To highlight the numerous ways that students can make connections, she offers a matrix of four different possibilities, starting with the difference between transfer and integration. As she observes, students sometimes consciously make connections, which she describes as an act of integration. She limits the notion of transfer to situations where the connections are 
automatic. She also distinguishes between connections that are successful and frustrated. Successful connections refer to those that have been effectively sold to the audience, while frustrated connections are those that are not seen as valuable. Her most important contribution is the idea that transfer involves not just observing similarities between contexts but also using rhetorical strategies to "sell" those connections to an audience.

The weakness of this approach is the "connecting" and "selling" processes remain separate. While she acknowledges that a learner must "sell" a practice, she does not have a similar mechanism for how the learner notices the connection in the first place. These processes are deeply intertwined - in the process of learning a practice, an individual not only must attempt to convince the audience of his/her aptitude, he/she also must look to his/her environment for feedback. To understand what I mean, it might help to introduce what Schwartz, Bransford, and Sears describe as the difference between "transferring out" and "transferring in." When researchers look at what "transfers out," they are concerned with whether a student applies a skill learned in a particular context, such as studies that examine if first-year writing students transfer their skills to future disciplinary courses. In contrast, "transferring in" refers to how learners use their previous skillsdepending on how a person interprets the situation, he/she might draw not just from previous college courses but also from other contexts. The idea of "transferring in" shifts our focus away from applying skills toward how students make sense of their surrounding environment.

The problem with focusing on "transferring out" is that it makes the scope of our research too narrow. If we are only looking for a moment where students directly makes a connection with a particular course, we might miss how they are continually drawing on 
the skills learned throughout their lives. Transfer studies are often inconclusive because learners draw from a rich array of resources - a person might look to their previous composition courses when beginning a new project, but he/she is equally capable of turning to workplace experiences, parental advice, and peer activities. Looking for what "transfer out" is like trying to locate a tiny thread in this tangled web of resources. Even in the situation where researchers observe a student making a connection, the danger is that noting what transferred will come at the expense of figuring out how it was transferred. The entire point of transfer research is to elucidate the processes by which students use skills learned in previous contexts. We risk rendering these broader learning strategies invisible when we are mostly concerned with what students draw from a particular situation.

To illustrate this point with an example from Nowacek's work, she discusses one case of "successful integration" where a student called upon a theory learned in a history course to write a literary analysis of Doctor Faustus. As she explains, "this act of transfer is best understood not as a matter of simple application; it is instead a more complex act of transfer-as-reconstruction, one that provided not just a brick but the entire blueprint for his argument" (44). Unfortunately, her analysis doesn't go much further than simply noting that the connection was crucial. Perhaps the reason the student made the connection was because of the similarities between history and literature courses—after all, knowledge is often shared across both disciplines, and they often deal with similar texts. We might also argue that they have a similar relationship with truth. In both disciplines, textual interpretation is a highly prized skill, and knowledge is rarely viewed as a fixed commodity. Nevertheless, a number of differences likely existed between his 
history and literature courses, which might include significant things like the rhetorical strategies expected by the instructors or minor things like the physical arrangement of the desks. Of course, what complicates this picture even more is that our perception is irrelevant. The student is the one that must make these observations. Given that is the case, we might take into account a number of other issues, such as whether he had successfully employed a similar strategy in previous literature courses. The point is that while we can certainly say something transferred in this situation, we are left without many clues as to how the student went about making the connection.

The difficulty partially stems from the metaphors that Nowacek uses to imagine the transfer process. As she explains, the "integration" metaphor "provides a compact and powerful way of understanding transfer as a rhetorical act, one that involves seeing and selling" (35). While selling is definitely a complex rhetorical act, seeing is often perceived as a more passive process. We rarely think that we have much control over our vision, aside perhaps from the ability to bring one object into focus while allowing the remaining elements to fade into the background. Following Schwartz et al., I want to suggest that the concept of interpretation offers a better model for how students go about making sense of the surrounding environment. Interpretation involves an act that is attentive to not just what is in the foreground but also what is hidden from view. In addition, while seeing is frequently imagined as an instinctual process, interpretation is considered a learned behavior that varies among members of different social groups.

As a way of illustrating what they mean by interpretation, Schwartz et al. discuss an unpublished experiment designed by Kay Burgess that examined what strategies different leaners use to tackle problems outside their realm of experience. She confronted 
both fifth-graders and college students with the difficult problem of how to develop a recovery plan for stimulating the growth of the bald eagle population in their state. The students began the study with little knowledge of the subject. In the first segment of the experiment, she directly asked how they would resolve the issue, and not surprisingly both groups failed to devise a workable solution. In contrast, the second part of the study attempted to discern what questions students would ask to obtain the information needed to tackle the problem. Some significant differences existed between the two groups. The fifth-graders mostly focused on specific characteristics of the eagles, such as their size, eating habits, and habitats. In contrast, the college students generally looked at the relationship between the eagle and their larger environment. The experiment was repeated among school principals, except this time she asked the principles to describe how they would learn to solve the problem. She discovered a similar phenomenon—once again, they were unable to determine a workable solution, but they had a better understanding of how to find an answer. They were much less confident about the usefulness of their prior knowledge and more willing to abandon their initial assumptions. They also considered issues that were mostly neglected by the students, such as how to build support for eagle recovery efforts in the larger community.

A number of lessons can be learned from the experiment, the most important being the enormous range of strategies that the study's subjects employed to develop a solution to the problem. A traditional study that looked entirely at what "transferred out" from biology courses might be disappointed at how far off all the learners were from actually developing a suitable recovery plan. However, when we look at what "transferred in" from their previous experiences, we discover that each subsequent 
generation had more nuanced methods for approaching the problem. The principals drew not just from their biology background but also from their professional experiences, where building strong social networks is necessary for any kind of action. In addition, the study demonstrates that learning a practice requires more than just developing a set of skills that will then be applied to similar situation in future contexts. Rather, we must also develop flexible methods of interpretation, so we are able to recognize our limitations when faced with new problems and start working toward developing innovative solutions.

Like many researchers, though, Schwartz et al. focus almost entirely on how the individual responds to the environment, which neglects the role of social groups in the transfer process. While people are continually experimenting with new approaches, no guarantee exists that these innovations will be seen as valuable. They must also be able to "sell" these practices to other practitioners. One way of imagining this process is through the idea of "developmental coupling." Drawing from phenomenology, King Beach derives the term through an analogy with the theory of co-evolution, which is used to describe the changing relationship between a species and its environment. As he explains, "the coupling itself is the primary unit of study and concern rather than the individual or the activity ... the coupling assumes that individuals move across space, time, and changing social activities, rather than being hermetically situated within an unchanging context" (47). To put this into Bourdieu's language, a practice refers not just to a set of cognitive habits but rather to the "coupling" that binds together the habitus and the field (see Figure 4.1). The most important part of Bourdieu's framework is the central role played by temporality. Rather than viewing students as moving between isolated 
contexts, they are depicted as on a trajectory through social space, whose motion is represented by the diagram's twisting arrows. A practice is not just a set of discrete skills — such as the ability to write complete sentences - but also the capacity to improvise these skills to meet the demands of the changing world.

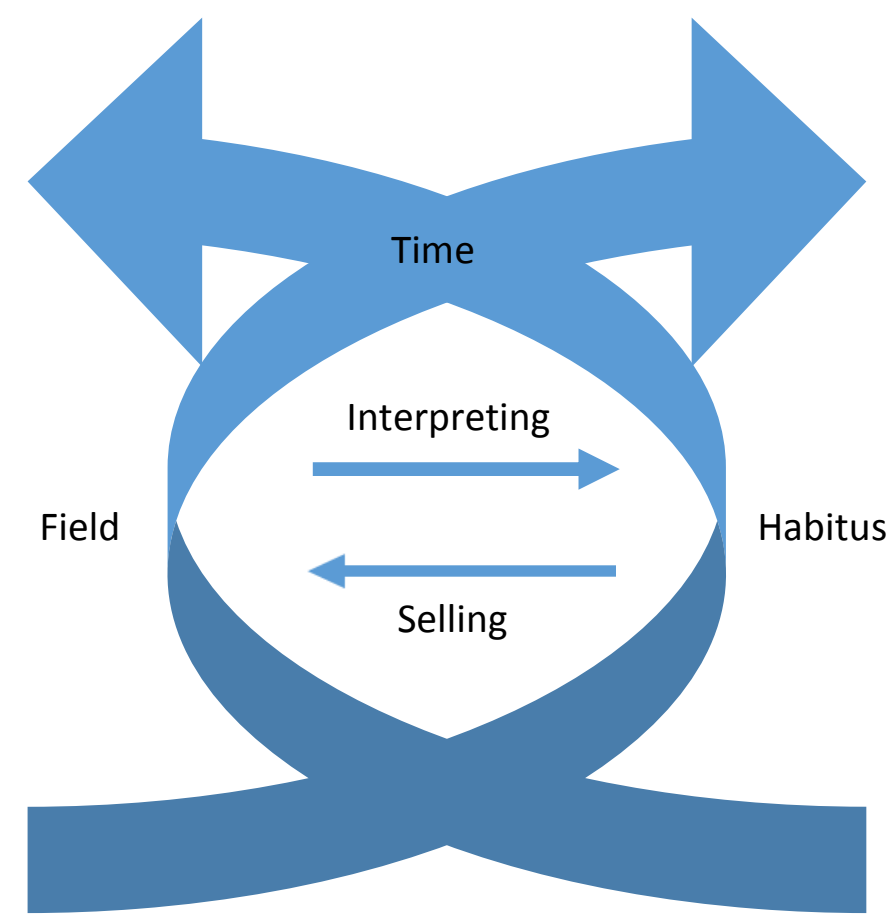

Figure 1. A Model of Practice

One of the reasons that Bourdieu was charged with determinism is that he never brought much clarity to the processes that underpin the act of improvisation. While he clearly felt that the field and habitus were undergoing continual transformation, he rarely articulated what brought about these changes. One metaphor that he uses in a couple instances is the idea of the hysteresis effect, a term that refers to the reversal of magnetic poles and its resulting effects. He wanted to emphasize that the field can shift its trajectory before the habitus has adjusted to accommodate the changes (Distinction 142; 
Invitation 130). The idea is that practitioners will continue to operate using their old musty cognitive dispositions even though they no longer reflect the field's current structure. He argued that people paradoxically cling even more tenaciously to their outdated practices even though they are in conflict with the social world. A contemporary example might be American conservatives who have come to argue even more stridently for the immorality of homosexual relationships. While we definitely can describe some social changes as being the product of something like the hysteresis effect, the concept seems to capture only a narrow range of situations. Even then, it primarily focuses on how the field transforms the habitus, which seems to not provide much clarification to how individual practitioners transform their social environment. The advantage of actororiented transfer theories is that they help clarify the improvisation process, especially the role played by our interpretations of the social world and the strategies we use to sell our practices. The twin arrows in the center of the diagram illustrate how interpreting and selling help maintain the reciprocal relationship between the two structures.

\section{The Virtuosity of Practice}

The notion that improvisation is at the heart of practice brings into question many of transfer theory's assumptions. Many researchers are committed to the notion that improvisation is a special event, which is partially why Salomon and Perkins distinguish between "transfer" and "mere learning." As they argue, "mere" learning happens when the situations are so similar that the application of prior skills is automatic, while the concept of transfer is generally reserved for moments where "learning has a side effect we were not perfectly confident it would have" (116). One way they reinforce this distinction is by arguing for the importance of conscious reflection. In their discussion of 
the distinction between the "low road" and "high road" transfer mechanisms, they emphasize "low road" transfer is a mundane occurrence that happens in situations that are so similar that the process is "quite painless and automatic" and requires little deliberate effort (117-18). In contrast, "high road" transfer between extremely different situations requires a more explicit act of formulating a mindful "abstraction in one situation that allows making a connection to another" (118). They seem to view moments of significant insight as being mostly the product of high road transfer, which is why they highlight the Gutenberg's invention of the printing press and Darwin's discovery of evolution as an example of this phenomenon.

The privileging of conscious reflection can also be seen in the recent calls for first-year composition to focus on "rhetorical awareness" rather than teaching particular academic genres. As Bergmann and Zepernick argue, focusing on rhetorical awareness should "help students recognize that they are making choices, and how to make those choices consciously" (142). Devitt's argument for encouraging students to develop "genre awareness" makes these claims even more explicit, to the extent that she states that "conscious awareness of anything makes mindful living more possible that it would be otherwise." Her hope is that students can use their genre awareness to avoid "following formula blindly because they will always be wondering about the contexts behind the forms" (202). In a similar fashion, Beaufort argues that increasing "mindfulness" among students is one of the main ways we can encourage the transfer of writing skills (152). Much like Salomon and Perkins, these authors are committed to the distinction between "blind" unconscious processes and "mindful" ones that are done with conscious awareness. The idea is that people who rely on automatic processes are trapped within 
mindless formulas and are less capable of questioning the ideologies circulated by various academic genres. As Nowacek argues, the problem with this position is that "meta-awareness" doesn't seem to be a necessary condition for transfer, particularly given the number of students in her study that seem to make interesting connections between disciplines without much conscious reflection. One of her students seemed quite capable of intuiting "the differences in the goals of the various disciplines he was being asked to negotiate" and was continually puzzled by his success (123). As she argued, focusing so much on "metacognitive abilities is tantamount to pointing to a black box in which a general cognitive ability magically operates" (17). Essentially, it is just serving as another "general writing skill" that will supposedly help students in all their courses.

Bourdieu's model makes no attempt to differentiate between "mindful" conscious choices and "blind" automatic ones. For him, improvisation is how people cope with the changing world, and it happens at all levels of awareness. Drawing an analogy with someone playing a sport, he argues that a person engaging in a practice should be viewed like someone who has a "feel for the game." The player must attend to not just "what he sees but to what he fore-sees ... he passes the ball not to the spot where his team-mate is but to the spot he will reach" (Logic 81). Much like a boxer, "each move triggers off a counter-move, every stance of the body becomes a sign pregnant with a meaning that the opponent has to grasp while it is still imminent" (Outline 11). Because we are caught up in the flow of time, other people in our environment are continually responding to our behaviors, adjusting their perspectives, and presenting us with new challenges. Our ability to thrive therefore does not come from repeating the same old patterns. We must also be capable of anticipating other people and choosing the best possible response. 
While conscious reflection is obviously one tool that we have at our disposal, our ability to improvise is intrinsic to practice and often happens without our active awareness. In Bourdieu's view, a person can't be said to have "learned" a practice until he/she figures out how to improvise.

While Bourdieu grants improvisation a central role, he also emphasizes that practices are bound in a number of ways, just not in the sense of a tacit "rule" that prevents people from engaging in certain actions. The first way that practices are constrained is by the brute fact of their temporality. At a certain point in an individual's history, he/she was taught their practices by other people, which caused the formation of a set of dispositions that influence his/her future action. Bourdieu argues that the main advantage of the concept of habitus is that it helps us move beyond the view that social agents are either entirely determined by external causes or "isolated monads" completely responsible for their own actions. They are the "product of history, of the history of the whole social field and of the accumulated experience of a path within the specific subfield" (Invitation 136). Everything from our parents to the experience of growing up in a particular community influences our trajectory. But, while our past constrains our practices in numerous respects, our future is also limited by other people, which is part of the reason that a practice must always be viewed as a "coupling" between the individual and the larger social environment. The field also has a history and is traveling upon its own trajectory, and its members have developed their own strategies for dealing with the world. As Turner observes, the reason people are able "to interact more or less successfully with the 'members of the community' ... is a matter of habit, a matter of having successfully responded to feedback and having developed habits of response that 
enable, among other things, the person's various purposes to be achieved" (75). The only way for individuals to achieve their goals is to take into account the feedback of other people. For that reason, we don't need to resort to any mysterious transcendental entities to explain the persistence of practices. The fact is that human beings are inherently social creatures - we start interacting with others from the moment we are born, and their influence provides the momentum that guides our subsequent movements through social space.

But the most important part of his model is how it shifts our understanding of the learning process. The problem with transfer theory is that it inevitably painted an unflattering picture of students. We became extremely concerned with whether students were "blinded" by their writing practices, to the extent that we questioned whether they were even capable of bringing what they learned from freshmen composition into other academic courses. Rather than introduce students to a wide range of practices with the idea that it would broaden their horizons, we began focusing narrowly on just teaching what we viewed as the particular set of routines they needed for the workplace. The most important question was "what" transferred between courses, and we discovered much to our dismay that it was very little—students were "strangers in strange lands" who seemed to move between each course completely clueless about where to begin the writing process. When we begin to look at things through Bourdieu's lens, people no longer seem like victims under the sway of the "rules" that they internalize from the social environment. Virtuosity is not a quality unique to a certain group of people but rather a quality that defines all practice:

Only a virtuoso with a perfect command of his 'art of living' can play on 
all the resources inherent in the ambiguities and uncertainties of behaviour and situation in order to produce the actions appropriate to each case ... . we are a long way, too, from norms and rules: doubtless there are slips, mistakes, and moments of clumsiness to be observed here as elsewhere; and also grammarians of decorum able to state (and elegantly, too) what it is right to do and say, but never presuming to encompass in a catalogue of recurrent situations and appropriate conduct, still less in a fatalistic model, the "art" of the necessary improvisation which defines excellence (Outline 8).

Because people are always transferring skills to grapple with new challenges, the concern here is no longer with what transferred between contexts. Rather, the question becomes how the virtuous that enter our classrooms use their understanding of the social world to improvise their writing practices.

\section{Final Thoughts}

Critics of first-year composition have often characterized it as an unusual course that is designed to provide "general" academic writing skills rather than instruction in a particular genre. Unfortunately, a vast gulf separates many academic disciplines, which makes it difficult to see what "general" skills would apply to all these contexts. Even if we wanted to align our courses directly with the genres that students will need in the future, we have no way of accommodating the writing done in every academic discipline. As I have been arguing, the problem with this claim is that it could be applied to all learning situations. At first glance, a course like business writing might seem to have a more definable content—-however, after students leave college, they will move forward 
into a vast array of workplaces, each with their institutional cultures and their own established set of procedures. The mere difference between school and the workplace will require some kind of adjustment. In fact, students must continue to modify their practices to the meet the needs of the changing workplace. When we are teaching a practice, we are not just helping students "learn genres." We are also introducing students to the process of how to improvise their writing practices. In the final chapter, I look more closely at strategies we can use to investigate how students go about navigating the "unexpected situations" that they encounter as they move throughout the academic curriculum and transition into the workplace. 


\section{CHAPTER V}

\section{DIRECTIONS FOR FUTURE RESEARCH}

The main problem with the existing transfer research is that it only explores a limited range of phenomenon. In one of Salomon and Perkins' paradigm examples of transfer, they discuss a fictional individual who grows frustrated with his inability to control his spending habits ("Rocky" 118). Looking back on his experiences, he remembers a strategy that his mother taught him for dealing with his childhood temper tantrums, where he was told to count to 10 in order to control his emotions. He applies this strategy to his shopping habits and discovers it helps control his impulses. In their description of his problem-solving process, they argue that he abstracted a principle from his previous experience that helped him deal with the situation. The problem with the explanation is that it focuses on the connection made by the shopper at the expense of other factors. Ultimately, the fact that he made a connection is not that significant, mainly because we're continually transferring skills from our previous experiences. Part of what it means to be a temporal being — an agent that operates within a fluctuating world —is having to respond to new challenges, whether it be as simple as adjusting to a driver drifting into our lane or as complex as writing in an unfamiliar genre. While it's necessary to note the connections being made by students, the more important consideration is how the individual responds to the social environment, and, perhaps even more importantly, how the social environment responds to the individual.

Rather than view "transfer" as some sort of special act, my argument throughout 
the dissertation has been that it is a fundamental part of practice. We must necessarily draw on our previous experiences to tackle new challenges. However, even though we are continually devising innovative solutions to problems, not every approach ultimately helps us achieve our goals. Our mission as composition researchers should be to understand the consequences of adopting certain strategies. Unfortunately, the problem is complicated by the fact that we can't assume that what students consciously report is an accurate reflection of their practices. As Bourdieu argues, innovation occurs at all levels of awareness, and some of our most profound insights might happen without our explicit knowledge. In addition, while it is tempting to look primarily at individual students, we can only fully assess the development of their writing skills by looking at their relationship with other people. As I discuss in the previous chapter, a practice is precisely the reciprocal relationship between a person's habitus and his/her social environment. Students are in the continual act of interpreting their surroundings and innovating their practices in response, and, at the same time, they are engaged in the act of selling their practices, which influences not only how other people perceive the individual but also their world.

This final chapter discusses the implications of this perspective for future composition research. While I don't have any easy solutions to the methodological problems outlined in the previous chapters, I suggest we should begin by investigating how students interpret their writing assignments. The existing transfer research offers some insight into what we might find, with many studies suggesting that students' are significantly influenced by their understanding of the politics of the university. Drawing particularly from research into naturalistic decision-making processes, I recommend 
focusing on what students perceive as "unexpected" tasks, which are of interest because they might provide a glimpse into what makes a student decide to improvise. However, we should be wary about importing other methodological approaches into our field, especially when it comes to how we go about interpreting interviews - rather than view them as an accurate descriptions of a person's actions, they should be seen as reflecting students' perceptions of their practices and what factors influence how they interpret the environment.

\section{Coping with the Unexpected}

The reason we became interested in transfer is because it seems like an intuitive way to explain how people deal with unusual problems. When people hit a snag during their typical routines, it makes sense that they will "transfer" skills from other areas to help deal with it. Of course, the problem is particularly acute for composition professionals, mainly because we teach such a wide range of generalist courses. Students that take introductory writing courses will be producing texts that seem much different than what they will be asked to write in their majors. On some basic level, all disciplines utilize similar writing skills, such as working with academic sources, developing transitions between paragraphs, and producing compelling arguments. However, the differences seem quite pronounced when we begin looking at the issue in more detail. Even those of us who teach professional communication courses must sometimes wonder whether teaching a business letter in a classroom setting is in any way similar to doing so in the workplace. Ultimately, we can't fully predict the situations that students will encounter in the future, but we know that they will differ considerably from than the assignments they complete in our courses. And, given the continual demand to justify our 
courses to administrators, we naturally have some anxiety about whether our pedagogy has any use to students in other disciplines.

While our impulse to look at how students handle unexpected situation is the right one, the problem is that we've mainly been looking for what students transfer rather than how they go about deciding what to transfer between courses. Our approach has almost been like looking at the raw materials used to build a sculpture rather than examine the final creation. Obviously, when a sculptor has a vision about what they want to create, they will utilize whatever they have available to make that vision a reality. The interesting question is not so much the raw materials but rather how the person went about deciding what to sculpt. The creation will ultimately transform the initial materials into something new-indeed, when we're talking about what it means to be an "expert," we typically mean someone who does more than just mechanically follow the same patterns (Dreyfus and Dreyfus 30-36). They need to be able to move beyond the limitations of their existing practices and respond dynamically to changes in the surrounding environment.

As Schwartz et. al claim in "Efficiency and Innovation in Transfer," the problem is that educational researchers have privileged what they view as efficient practices, which they define as those that allow people to "rapidly retrieve and accurately apply appropriate knowledge and skills to solve a problem or understand an explanation" (28). Their paradigm example of what they mean by efficiency is doctors who have become so familiar with a medical procedure that they can almost immediately formulate a diagnosis upon seeing a new patient (28). While each patient is to a certain degree different, experienced doctors have encountered such a wide range of ailments that they are able to 
quickly determine which details are relevant and use them to make a decision. For that reason, when we are looking for someone to solve a problem, the first question we inevitably ask is how much experience people have in the area, largely because we know the importance of being continually confronted with certain situations in the development of expertise. While focusing on this dimension of practice is certainly important, the problem is that the world is constantly in flux, which means people most frequently will stumble into unexpected situations. It is certainly dangerous to have a surgeon who doesn't have enough practice with a procedure, but it is perhaps even more dangerous to have one unable to recognize the limitations of a particular approach, especially when he/she is in the middle of a dangerous operation.

For that reason, Schwartz et al. argue that we must also appreciate the role played by innovation, which they characterize as the ability to "rearrange their environments or their thinking to handle new types of problems or information" (30). One of the advantages of their approach is that they recognize the importance of developing efficient routines in stimulating innovation. After we have developed a set of procedures that we can execute in a wide range of situations, we are more capable of having our intellectual resources free to notice difficulties in the process. They argue that one essential component of innovation is the recognition of our own limitations. They highlight a study by Samuel S. Wineburg that presented both history experts and college students with a set or unfamiliar history problems involving Abraham Lincoln. Interestingly, the history experts were less likely to make questionable assumptions about the nineteenth century, while the college students made flawed claims based on their contemporary understandings (30). Schwartz et al. argue that what triggers innovation is frequently the 
recognition that the current procedures are failing and therefore a different approach might be appropriate (32). Jean Lave makes a similar argument when she points out that grocery store shoppers only initiate problem solving procedures when they hit a "snag" in the flow of an activity (165-168).

The difficulty is how to go about studying such issues, mainly because the effect our pedagogy has on helping students handle "snags" doesn't always have an immediate effect. If we teach students to write in a particular genre, we can immediately see its impact when we ask them to produce something similar in a future assignment. We can see how helping a student write a resume is useful when we hear back from students that they used it to get a job. Teaching students how to improvise, on the other hand, doesn't produce easily measurable results. The problem is rendered more complex because the most obvious method for examining decision-making processes — which is to simply ask people how they go about handling problems - raises numerous concerns, especially in regard to whether we are getting an accurate glimpse into an individual's practices or simply their post-hoc rationalizations. Research into memory shows that the mere act of recalling an experience can actually change how a person remembers it (Siegel 42). And, as Bourdieu argues, the simple fact that the practitioner must explain their decision to an "outsider" means that they must assume a pedagogical stance when describing the practice (Outline 19). Nevertheless, interviews play an important role in any examination of decision-making process, so long as they are situated within a more comprehensive understanding of practices.

In Sources of Power: How People Make Decisions, Gary Klein discusses the results of a series of studies that rely almost entirely on interviews, and his results 
illustrate a number of facts about human behavior while also pointing to some of the limitations of relying on introspection. His research began upon winning a military contract to examine how firefighters make tough decisions. Klein entered the study speculating that firefighters would operate under what he described as the "two-option hypothesis": essentially, they would have one option that seemed intuitively obvious, and they would have an alternative that seemed to be a less attractive choice. He discovered through his interviews that they didn't even seem to be making what he considered “decisions," in they didn't rationally consider multiple options and then select the best choice. They reported immediately taking a course of action, and when the situation changed and that option was no longer available, they seemed to automatically know how to adjust their behaviors. In fact, it seemed to be novices who were the ones that needed to carefully consider the advantages of several competing choices. In a dramatic example, he discusses an instance where rural firefighters needed to contain an enormous blaze at a farm of oil tankers. Due to their lack of experience with such large fires, they ended up proceeding with a scattered mish-mash of different approaches, until they final decided to stop trying to contain the fire and devote all their energies to thinking through their options. Interestingly, their deliberations by themselves never produced a workable solution —instead, they ended up calling in a team of experts from outside the fire department who ended up using large amounts of foam to solve the problem (21-23).

His research focuses on what he describes as "naturalistic decision-making settings," by which he means studying how people make difficult choices during the course of their actual lives. Rather than present people with a pre-defined task, he wants to understand how they deal with messy problems, ones that involve time pressure, high 
stakes, unclear goals, and inadequate information. One of the important features of his methodology is his concern with exceptional cases. Rather than concern himself with abstract generalities about the workplace, he wanted his subjects to discuss their most memorable experiences (12). As he explains, "By focusing on the nonroutine cases, we were asking them about the most interesting ones - the ones they come back to the station house and tell everybody about" (10). The researchers then searched through their stories for "decision points," which are moments where a choice was made in the face of other reasonable alternatives. Afterwards, they constructed a timeline of the event based on the interviews.

The most obvious problem is that he interprets the stories being told by the practitioners as accurate representations of their experiences. While his team sometimes "observes decision makers in action," he admits that his research relies almost exclusively on introspection (291). He confesses that he has no way of knowing "if the things [his subjects] are telling us are true, or maybe just some ideas that they are making up" (291). Of course, the question is not if people are "making things up" but rather how we go about interpreting their memories. Looking at just the example of firefighters, they might be influenced by popular representations of their job, where people go charging heroically into blazes. The stories also likely have a rhetorical component, in that they are intended not just to report the "truth" about an experience but also to sell people on a particular image of their profession. What researchers discover through an interview is not necessarily "what actually happened" but rather people's perceptions of the event.

For the purposes of composition studies, though, one of our most important considerations is how people are interpreting situations and selling their practices. Rather 
than see people's stories as accurate representations of events, we need to view them as illustrations of how people are mapping out their social environment and understanding the relationships that hold between people. Furthermore, the reason to focus on exceptional cases is not necessarily because they are the most "interesting" ones but rather because they are the moments where people had to adjust their interpretation of events. The reason people remember these experiences vividly is that they are often accompanied by emotions, which psychologists argue are more likely to be classified as "important" by the brain and therefore recalled more easily in the future (Siegel 47). As Nowacek points out, transfer has an affective component—in the process of making connections, students experience various emotions, often cycling between frustration and elation (27). These emotions should be taken as indicators of the importance of an experience. Even though we have frequently interpreted our students' sense of frustration as "negative transfer," the fact is that frustration is a part of the learning process, and we need to accept that student will be frustrated before they ultimately decide on what they see as the right approach.

Unfortunately, the transfer research only offers a few glimpses into how students deal with unfamiliar situations. In one example, Nowacek describes a case where a student at a Catholic university was asked to write an essay on Thomas Aquinas. Many students reported having difficulties with the assignment, mainly because the teacher was asking them to do something new. He was asking students not to criticize Aquinas but to articulate his theological project. As the teacher explained in the assignment prompt, he wanted students to "recreate the way in which, according to Aquinas, human beings achieve salvation ... to make a case for the reasonableness of Aquinas's theology on this 
issue. Be his defense lawyer" (112). While the task was confusing for many students, it is nonetheless quite typical in the humanities, where it is important to understand the complexities of certain foundational figures. Interestingly, though, the student didn't see the professor as just asking students to understand the ideas of a difficult theologian. As a Quaker attending a Catholic institution, she was self-conscious about her own religious identity, so she viewed the assignment as coercive. She interpreted the professor's demand to "get inside Aquinas's mind" as a subtle attempt to instill Catholic beliefs in the students.

Because she interpreted the assignment as an attempt at proselytization, she spent a considerable amount of time figuring out a way she could circumvent the assignment to discuss her own views. She wanted to contrast her Quaker beliefs with what she saw as the dominant perspective of her Catholic institution. After some reflection, she decided to make the choice of writing the essay as a dialogue between Aquinas and a "highly deferential student" (57). While the approach was unorthodox, she sought to meet the assignment requirements by "getting into the head" of Aquinas through his fictional representation while still being able to challenge his theological positions. Once again, the interesting part about this example is how incidental her "connections" were to solving the problem. While she likely never would have considered writing a philosophical dialogue if she had never encountered the genre in a previous philosophy class, the driving force behind her approach was the perceived identity conflict. She would have unquestionably tried to pull from other resources even if she had never encountered the dialogue genre. 
The interesting question is what made the student feel that the instructor had a religious rather than a disciplinary agenda. The other students didn't seem to view him as attempting to promote his Catholic beliefs. When the Quaker student suggested that the instructor had a religious agenda in a focus group interview, the other students responded, "I didn't see it that way at all" (56). And, while other students struggled with the assignment, they interpreted their task in a different fashion - another student who wrote what the professor viewed as a successful paper paraphrased the assignment as asking "what the guy's project is" (119). The instructor himself portrayed the assignment as a "critical exercise" that demanded the students contemplate the following questions: "What ties [Aquinas's] system together? What's the most important thing about it?" (112). Surprisingly, the Quaker student ultimately did well on the assignment even though her interpretation seemed at odds with what the instructor presented in the classroom. At least on a certain level, she managed to sell her understanding of Aquinas even though she wrote in an unconventional genre, although hearing the professor's thoughts on the issue would have added more clarity to whether he thought she had achieved the assignment's objectives.

The previous example highlights the central role played by the student's understanding of the politics of the university in interpreting their writing assignments. In this particular case, the student viewed the university's Catholic mission as more significant than other pedagogical concerns. Another question is how the student's perception of the instructor's political affiliations - whether they are "liberal" or "conservative" - influence how they interpret their assignments. However, perhaps even more important is how students understand both the academic disciplines and the power 
relationships that hold between them. One of the student's difficulties in completing the Aquinas assignment is her lack of understanding of what it means to "do theology"rather than view analyzing Aquinas as an academic exercise, she interpreted it through the more familiar framework of popular religious debates, where Aquinas is not only a research interest but the foundation for a set of Catholic doctrines. In the same fashion, our students come to the classroom with a set of perceptions of what it means to "do English," which are established not just by popular culture but also by high school experiences. They may find it hard to dislodge certain views when they have spent years completing grammar exercises, writing personal narratives, and reading literature. These perceptions also might be reinforced by professors across the disciplines, who might find it useful to contrast their own writing assignments with the "creative" exercises that they imagine are happening in the English department. And, of course, English is a conflicted discipline, to the extent that students might enter two introductory writing classes and receive entirely different experiences. All these factors might make it difficult for students to see how their English classes are relevant to their education.

The transfer research offers some insight into how the English department is perceived by students. In Bergmann and Zepernick's series of focus groups with students, they discovered that they tended to associate English with "flowery" and "creative" prose, which they saw as distinct from the more academic work done in other disciplines. Of course, the fact that their university is considered the "science and engineering campus of its statewide university system" might have impacted the results, perhaps because students from the science have a more negative view of the English department than those in other disciplines (143). On the other hand, even though the subjects in Lee 
Ann Carroll's Rehearsing New Roles: How College Students Develop as Writers come from a variety of backgrounds, they expressed similar views. The longitudinal study included both interviews and writing samples collected throughout the student's college education. One accounting student argued the essay topics in her introductory English course were "random," and she thought successful English essays required considerable “fluff” (66). Another student was asked to read Cornel West's Race Matters, and she expressed frustration with having to "fight" the professor's left-leaning agenda. At the end of her education, she acknowledged that she learned something from the course, but she expressed dismay at knowing that she would receive poor grades for disagreeing with his perspective (68). These studies point to the need to have a better understanding of how the English department is perceived across other disciplines. On a basic level, we need a better sense of how students imagine the English department and how their perceptions affect their performance. However, we also need to consider how their perspective is influenced by other college professors and past educational experiences.

More importantly, we need more information about how different pedagogical approaches prepare students for uncertainty. At the moment, we have only a few concrete examples of students dealing with such situations, and they are scattered across a disconnected set of studies on transfer and writing development. While we might speculate that the politics of the university plays a significant role in how students interpret assignments, the truth is that we don't fully know how students handle what they perceive as an "unusual" task. To be quite honest, we don't even know what causes them to interpret an assignment as being "unusual" or how capable they are at recognizing their limitations. We also don't know the role played by metacognition when 
students stumble across "snags" — while some scholars have argued that metacognition is the key to helping students deal with unexpected situations, the research gives us some reason to be skeptical (Nowacek 142). In many respects, "metacognition" is a concept that brings us dangerously close to the "rational actor theories" that Bourdieu so bitterly opposed throughout his work (Invitation 124). While simply interviewing students is a good place to start such research, we ultimately need to go beyond such things to include observations of their actual writing practices_- perhaps by looking at how their description of their processes match up with their changing drafts—and how their professors end up viewing the quality of their finished products.

\section{Conclusion}

Much of the impetus behind recent transfer studies is that our knowledge about how students develop as they move throughout the curriculum is woefully inadequate. In “Teaching about Writing, Righting Misconceptions: (Re)Envisioning 'First-Year Composition as 'Introduction to Writing Studies,"” Douglas Downs and Elizabeth Wardle make a provocative claim about the current state of transfer research, arguing that many introductory courses rely on the "deeply ingrained assumption with little empirical verification" that writing practices transfer between different situations. When describing the state of knowledge of our discipline, they rather bleakly state that we don't know "what genre and tasks will help students in the myriad writing situations they will later find themselves. We do not know how writing in the major develops" (557) Without more research, they believe teachers will be at a loss to figure out what to teach in introductory courses, mainly because it will be impossible to tell if "choosing genre A or genre B will be of service to students who must write genre B or genre C later on" (577). 
They argue that our ability to design effective courses will be crippled if we don't investigate these issues.

However, when we look at the actual literature, we find it provides us with considerable insight into student writing practices. We encounter students raising critical questions about what ideologies are promoted by the university. We see students utilizing knowledge learned in a wide variety of contexts to solve difficult problems. We watch students progress through their university education and slowly gain more rhetorical sophistication. We watch them struggle with unfamiliar genres and employ a variety of strategies to tackle them. Overall, we learn that even though students face a wide range of different situations, they find a way to muddle through them, sometimes in spite of the limitations of the pedagogy that they encounter in their previous courses.

One of my major concerns about "transfer theory" is that it causes us to overlook these insights and instead ends up making students "look dumb." If our focus is on whether students draw on a particular genre learned in an introductory writing course, we end up missing how they creatively draw on the totality of their experience. Effective writers need to have competency in a set of particular genres, but they also need to be able to address the particularities of the moment, which might seem to follow a familiar pattern but also might deviate from it in significant ways. One of the main questions we need answered is how students go about tackling these unexpected situations, and, even more importantly, how to teach them to effectively respond to them. What we've learned from the transfer research is that students will inevitably face situations we can't anticipate, whether it is in the workplace or in other courses, and part of what it means to be rhetorically sophisticated is dealing with such difficulties. While this doesn't suggest 
that we should just give up teaching students particular genres, we must acknowledge the world is in flux — not only is it impossible to predict the conditions that might exist in the workplace, we also can't know how other factors, such as the development of new technologies and communication techniques, will change what it means to "write" for future generations. The one thing we can know for certain is that students will need to deal with uncertainty. 


\section{REFERENCES}

Artemeva, Natasha. "A Time to Speak, a Time to Act: A Rhetorical Genre Analysis of a Novice Engineer's Calculated Risk Taking." Journal of Business and Technical Communication 19 (2005): 389-423. Print.

Bargh, John, and Ezequiel Morsella. "The Unconscious Mind.” Perspectives on Psychological Science 3.1 (2008): 73-79. Print.

Bawarshi, Anis S. Genre and the Invention of the Writer: Reconsidering the Place of Invention in Composition. Logan: Utah State UP, 2003. Print.

Beach, King. "Consequential Transitions: A Developmental View of Knowledge Propagation Through Social Organizations." Between School and Work: New Perspectives on Boundary-Crossing. Ed. Terttu Tuomi-Gröhn and Yrjö Engeström. Boston: Pergamon, 2003. 39-61. Print.

Beaufort, Anne. College Writing and Beyond. Logan, UT: Utah State UP, 2007. Print.

Bergmann, Linda, and Janet Zepernick. "Disciplinarity and Transfer: Students' Perceptions of Learning to Write.” WPA: Writing Program Administration 31 (2007): 124-49. Print.

Bizzell, Patricia. "Cognition, Convention, and Certainty: What We Need to Know about Writing." Pre/Text 3 (1982): 213-243. Print.

Bourdieu, Pierre. Distinction: A Social Critique of the Judgement of Taste. Trans. Richard Nice. Cambridge, Mass: Harvard UP, 1984. Print.

---. Language and Symbolic Power. Trans. Gino Raymond and Matthew Adamson. 
Cambridge, Mass: Harvard UP, 1991. Print.

---. The Logic of Practice. Trans. Richard Nice. Stanford: Stanford UP, 1990. Print.

---. Outline of a Theory of Practice. Trans. Richard Nice. Cambridge: Cambridge UP, 1977. Print.

Bourdieu, Pierre, and Loïc J. D. Wacquant. An Invitation to Reflexive Sociology. Chicago: The U of Chicago P, 1992.

Canagarajah, A. Suresh. "The Place of World Englishes in Composition: Pluralization Continued." College Composition and Communication 57.4 (2006): 586-619. Print.

Carroll, Lee Ann. Rehearsing New Roles: How College Students Develop as Writers. Southern Illinois UP, 2002. Print.

Cooper, Marilyn. "Why Are We Talking about Discourse Communities? Or Functionalism Rears Its Ugly Head Once More.” Writing as Social Action. Ed. Marilyn Cooper and Michael Holzman. Portsmouth: Boynton/Cook, 1989. 20220. Print.

Cooper, Marilyn and Michael Holzman. "Talking about Protocols." College Composition and Communication 34.3 (1983): 284-93. Print.

Kalantzis, Mary and Bill Cope. "Changing the Role of Schools." Multiliteracies: Literacy Learning and the Design of Social Futures. Ed. Bill Cope and Mary Kalantzis. New York: Routledge, 2000. 121-145. Print.

Crowley, Sharon. Composition in the University: Historical and Polemical Essays. U of Pittsburgh P, 1998. Print.

Detterman, Douglas. "The Case for the Prosecution: Transfer as an Epiphenomenon." 
Transfer on Trial: Intelligence, Cognition, and Instruction. Ed. Douglas K. Detterman and Robert J. Sternberg. Norwood, NJ: Ablex, 1993. 1-25. Print. Devitt, Amy. Writing Genres. Carbondale, IL: S Illinois UP, 2004. Print.

Dias, Patrick, Aviva Freedman, Peter Medway, and Anthony Paré. Worlds Apart: Acting and Writing in Academic and Workplace Contexts. Mahwah, NJ: Lawrence Erlbaum, 1999. Print.

Downs, Doug and Elizabeth Wardle. "Teaching About Writing, Righting Misconceptions: (Re)Envisioning FYC as Intro to Writing Studies." College Composition and Communication 58.4 (2007): 552-84. Print.

Dreyfus, Hubert, and Stuart Dreyfus. Mind over Machine: The Power of Human Intuition and Expertise in the Era of the Computer. New York: Free Press, 1986. Print.

Fahnestock, Jeanne, and Marie Secor. "The Rhetoric of Literary Criticism.” Textual Dynamics of the Professions: Historical and Contemporary Studies of Writing in Professional Communities. Ed. Charles Bazermen and James Paradis. Madison: U of Wisconsin P, 1991. 77-96. Print.

Ford, Julie Dyke. “Knowledge Transfer Across Disciplines: Tracking Rhetorical Strategies from a Technical Communication Classroom to an Engineering Classroom." IEEE Transactions on Professional Communication 47.4 (2004): 301-315. Print.

Freadman, Anne. “Anyone for Tennis?” Genre and the New Rhetoric. Ed. Aviva Freedman and Peter Medway. London: Taylor, 1994. Print.

Freedman, Aviva. "Show and Tell? The Role of Explicit Teaching in the Learning of New Genres." Research in the Teaching of English 27 (1993): 222-51. Print. 
Giroux, Henry A. "Theories of Reproduction and Resistance in the New Sociology of Education: a Critical Analysis." Harvard Educational Review 53.3 (1983): 25793. Print.

Hammer, David, Andrew Elby, Rachel E. Scheer, and Edward F. Redish. "Resources, Framing, and Transfer.” Mestre 89-119.

Harris, John. "The Idea of Community in the Study of Writing." College Composition and Communication 40.1 (1989): 11-22. Print.

Horner, Bruce. Terms of Work for Composition: A Materialist Critique. Albany: State U of New York P, 2000. Print.

Jenkins, Richard. Pierre Bourdieu. London: Routledge, 1992. Print.

Klein, Gary A. Sources of Power: How People Make Decisions. Cambridge, Mass: MIT Press, 1999. Print.

King, Anthony. "Thinking with Bourdieu against Bourdieu: a 'Practical' Critique of the Habitus." Sociological Theory 18.3 (2000): 417-433. Print.

Lave, Jean. Cognition in Practice: Mind, Mathematics, and Culture in Everyday Life. Cambridge: Cambridge UP, 1988. Print.

Lave, Jean, and Etienne Wenger. Situated Learning: Legitimate Peripheral Participation. Cambridge: Cambridge UP, 1991. Print.

Lizardo, Omar. "The Cognitive Origins of Bourdieu's Habitus." Journal for the Theory of Social Behaviour 34.4 (2004): 375-401. Print.

Lobato, Joanne. "How Design Experiments Can Inform a Rethinking of Transfer and Vice Versa." Educational Researcher 32.1 (2003): 17-20. Print.

Luke, Allan. "Genres of Power? Literacy Education and the Production of Capital." 
Literacy in Society. Ed. Ruqaiya Hasan and Geoff Williams. New York: Longman, 1998. 308-38. Print.

Marton, Ference. "Sameness and Difference in Transfer Studies." The Journal of the Learning Sciences 15 (2006): 499-535. Print.

Mazis, Glen A. Humans, Animals, Machines: Blurring Boundaries. Albany, NY: SUNY Press, 2008. Print.

McCarthy, Lucille P. "A Stranger in Strange Lands: A College Student Writing across the Curriculum.” Research in the Teaching of English 21 (1987): 233-65. Print.

Medway, Peter. "Fuzzy Genres and Community Identities: The Case of Architecture Students' Sketchbooks." The Rhetoric and Ideology of Genre: Strategies for Stability and Change. Ed. Richard Coe, Lorelei Lingard, and Tatiana Teslenko. Cresskill, NJ: Hampton, 2002. Print.

Mestre, Jose, ed. Transfer of Learning from a Multidisciplinary Perspective. Greenwich, CT: Information Age, 2005. Print.

Miller, Carolyn R. “Genre as Social Action.” Quarterly Journal of Speech 70 (1984): 151-67. Print.

Nash, Roy. "Bourdieu on Education and Social and Cultural Reproduction." British Journal of Sociology of Education 11.4 (1990): 431-447. Print.

Nowacek, Rebecca S. Agents of Integration: Understanding Transfer as a Rhetorical Act. Carbondale: Southern Illinois UP, 2011. Print.

Perkins, D. N., and Gavriel Salomon. "The Science and Art of Transfer." The Thinking Classroom. The Cognitive Skills Group at Harvard Project Zero. 1999. Web. 30 Nov. 2014. < https://learnweb.harvard.edu/alps/thinking/docs/trancost.pdf $>$ 
Petraglia, Joseph, ed. Reconceiving Writing, Rethinking Writing Instruction. Mahwah, NJ: Lawrence Erlbaum, 1995. Print.

---. "Writing as an Unnatural Act." Petraglia 79-101.

Popken, Randall. “Genre Transfer in Developing Adult Writers.” Focuses 5 (1992): 3-17. Print.

Prior, Paul. Writing/Disciplinarity: A Sociohistoric Account of Literate Activity in the Academy. Mahwah, NJ: Lawrence Erlbaum, 1998. Print.

Rorty, Richard. Contingency, Irony, and Solidarity. Cambridge: Cambridge UP, 1989. Print.

Russell, David. “Activity Theory and Its Implications for Writing Instruction.” Petraglia 51-77. Print.

---. "Rethinking Genre in School and Society: An Activity Theory Analysis." Written Communication 14.4 (1997): 504-54. Print.

Salomon, Gavriel, and David Perkins. "Rocky Roads to Transfer: Rethinking Mechanisms of a Neglected Phenomenon.” Educational Psychologist 24.2 (1989): 113-142. Print.

Schwartz, Daniel L., John D. Bransford, and David Sears. "Efficiency and Innovation in Transfer." Mestre 1-51.

Siegel, Daniel J. The Developing Mind: Toward a Neurobiology of Interpersonal Experience. New York: Guilford Press, 1999. Print.

Smart, Graham, and Nicole Brown. "Learning Transfer or Transforming Learning?: Student Interns Reinventing Expert Writing Practices in the Workplace.” Technostyle 18.1 (2002): 117-141. Print. 
Smit, David. The End of Composition Studies. Carbondale, IL: Southern Illinois UP, 2004. Print.

Takayoshi, Pamela, and Cynthia Selfe. "Thinking about Multimodality." Multimodal Composition: Resources for Teachers. Cresskill, NJ: Hampton Press, 2007. 1-12. Print.

Thompson, Evan. Mind in Life: Biology, Phenomenology, and the Sciences of Mind. Cambridge: Harvard UP, 2007. Print.

Tomlinson, Barbara. "Talking About the Composing Process: the Limitations of Retrospective Accounts." Written Communication 1.4 (1984): 429-45. Print.

Turner, Stephen P. Brains / Practices / Relativism: Social Theory after Cognitive Science. Chicago: U of Chicago P, 2002. Print.

---. "Mirror Neurons and Practices: a Response to Lizardo." Journal for the Theory of Social Behaviour 37.3 (2007): 351-371. Print.

---. The Social Theory of Practices: Tradition, Tacit Knowledge, and Presuppositions. Chicago: U of Chicago P, 1994. Print.

Walvoord, Barbara and Lucille P. McCarthy. Thinking and Writing in College: A Naturalistic Study of Students in Four Disciplines. Urbana, IL: NCTE, 1990. Print.

Wardle, Elizabeth. “'Mutt Genres' and the Goal of FYC: Can We Help Students Write the Genres of the University?" College Composition and Communication 60:4 (2009): 765-80. Print.

---. "Understanding 'Transfer' from FYC." WPA: Writing Program Administration 31 (2007): 65-85. Print. 
Wenger, Etienne. Communities of Practice: Learning, Meaning, and Identity. Cambridge, U.K: Cambridge University Press, 1998. Print.

Wilder, Laura. "Get Comfortable with Uncertainty: a Study of the Conventional Values of Literary Analysis in an Undergraduate Literature Course." Written Communication 19.1 (2002): 175-221. Print. 


\section{CURRICULUM VITAE}

\section{KENNETH A SMITH}

628 Bermuda Lane

Louisville, KY 40213

\section{EDUCATION}

Ph.D. English — Composition/Rhetoric, University of Louisville, December 2014 Dissertation: "Transfer and the Commodification of Practice"

Committee: Joanna Wolfe (Chair), Min-Zhan Lu, Bruce Horner, Susan Ryan, and Marilyn Cooper

M.A. English - Composition/Rhetoric, Middle Tennessee State University, May 2007

Thesis: "Searching for the Big Picture: Toward an Assessment Model for Writing Fellows Programs"

Committee: Dr. Trixie Smith (Chair) and Dr. Allison Smith

B.A. English - Creative Writing, Minnesota State University Moorhead, May 2003

\section{CONFERENCE PRESENTATIONS}

"Building a Common Playground: Making Meaningful Gamification for Composition." With Barrie Olson, Stephen Cohen, and Robert Terry. The Watson Conference.

Louisville, KY. October 16-18, 2014.

"Beyond Gold Stars: Making Gamification Meaningful and Valuable for Students." With Barrie Olson, Stephen Cohen, and Robert Terry. The Conference on College

Composition and Communication. Indianapolis, IN. March 19-22, 2014

"Revising the 'Writing-about-Writing' Course: Developing Skillful Writing Learners through Course Document Analysis." The Conference on College Composition and Communication. Atlanta, GA. April 6-9, 2011.

"Facilitating Reflective Awareness through Written Comments." Watson Conference. Louisville, KY. October 14-16, 2010.

"Abolition Incarnate: William Lloyd Garrison and the Construction of Identity in the Liberator's 'Refuge of Oppression' column.” Rhetoric Society of America. Minneapolis, MN. May 28-31, 2010. 
"From Transfer to Negotiation: Examining the Uses and Limitations of the Transfer Metaphor.” Writing Across the Curriculum. Bloomington, IN. May 20-23, 2010.

"Transfer Into, Outside, and Beyond the First-Year Composition Classroom." With Alicia Brazeau. Computers and Writing. West Lafayette, IN. May 20-23, 2010.

"Rethinking Student Resistance in Peer Tutoring Programs." The Conference on College Composition and Communication. Louisville, KY. March 17-20, 2010.

"The Examined Life - Cyberspace Style: The Construction of Space in the \#philosophy IRC Undernet Community." Computers and Writing. Davis, CA. June 18-21, 2009.

“(Re)Designing (Our) Selves and Sequences - New Perspectives on First-Year Composition Course Development." With Lisa Arnold, Vanessa Kraemer, Laura Detmering, and, Brice Nordquist. College English Association. Pittsburgh, PA. March 26-29, 2009.

"Guerrilla Marketing Operations: Using In-Class Workshops as Advertising Space for the Writing Center." With Mark Rickert. International Writing Center Association. Houston, TX. April 13, 2007.

"From English Student to Knight Errant: Experiencing the Quest through Pen-and-Paper Role-Playing Games." With Adam McInturff, Wanda McInturff, and Jennifer Cain. The Seventh Annual Conference on Teaching Medieval Literature. Lawrenceville, GA. March 3, 2007.

“Tutoring Speech Students in the Writing Center." Southeastern Writing Center Association. Nashville, TN. February 10, 2007

"On Writing Good: Assisting Students with Humorous Prose in the Writing Center." National Conference on Peer Tutoring in Writing. Ann Arbor, MI. November 12, 2006.

"The Inverted Mirror: The Rhetorical Function of the Abject in the Dungeons \& Dragons Panic." Annual Conference of the Mid-Atlantic Popular / American Culture Association. Baltimore, MD. October 29, 2006.

"Working with Students in Content Area Classes." Tennessee Writing Center Collaborative. Murfreesboro, TN. October 7, 2006.

"The Impossible Triangle: The Relationship between Paradox and Doubt in The Canterbury Tales.” English Graduate Student Organization Re-Visioning Conference. Murfreesboro, Tennessee. April 29, 2006.

"Writing Fellows: Assisting Across the Disciplines." Tennessee Writing Center Collaborative. Clarksville, TN, October 7, 2006. 
"What We Learned as Wage Slaves: Relating to Students with Experience from Beyond the Writing Center." With Claire Bates and Emily Murray. Southeastern Writing Center Association's Annual Conference. Chapel Hill, NC. February 17, 2006.

\section{TEACHING EXPERIENCE}

Part-Time Lecturer, University of Louisville, 2012-Present

English 101, Introduction to College Writing

English 102, Intermediate College Writing

English 306, Business Writing

Graduate Teaching Assistant, University of Louisville, 2007-2012

English 101, Introduction to College Writing

English 102, Intermediate College Writing

English 311, American Literature I

Graduate Teaching Assistant, Middle Tennessee State University, 2006-2007

English 101, Expository Writing

\section{ADMINISTRATIVE AND PROFESSIONAL EXPERIENCE}

Professional Development Workshop Coordinator, University of Louisville, 2011-2012

Helped design a set of professional development workshops to bring our

curriculum in line with the new campus-wide critical thinking standards and the Common Core Curriculum standards; coordinated activities with the part-time faculty on subjects ranging from composing effective writing assignments to reconsidering commenting strategies.

Research Assistant, University of Louisville, 2010-2011

Prepared materials for Suzette Henke's graduate and undergraduate literature courses; edited bibliographies and book manuscripts; designed recruitment materials and co-organized informational sessions for the new 2011 London Program, a study abroad opportunity that was being offered for the first time by the English department.

Assistant Director of the Writing Center, University of Louisville, 2009-2010

Tutored students in the writing center from diverse fields and backgrounds, ranging from engineering students to freshmen in first-year composition; conducted and designed workshops on a variety issues for university staff members and disciplinary classes; mentored and conducted evaluations of incoming tutors; helped lead a classroom session in the graduate writing center theory course; designed and conducted a small assessment project; developed writing center resources, including handouts and workshop materials.

Teaching Assistant, University of Louisville, 2008-2009

Assisted Suzette Henke with Medical Narrative, a literature course for fourth-year students in the University of Louisville's nationally recognized medical school; 
designed PowerPoint presentations for weekly lectures; assisted with class

discussions; graded and provided feedback on student essays.

Graduate Student Administrator of the Writing Center, Middle Tennessee State University, 2006-2007

Coordinated the Writing Fellows program, a WAC initiative that placed writing center tutors into communications, physical science, music, and women's studies classes; served as a mentor for new writing center tutors; conducted evaluations of tutors; maintained and developed writing center resources, including handbooks, workshops, and handouts.

Writing Assistant, Middle Tennessee State University, 2005-2006

Tutored students from diverse fields and backgrounds, ranging from graduate students in health education to freshmen in expository writing; conducted and designed workshops on MLA style, essay organization, and peer revision.

Writing Fellow, Middle Tennessee State University, 2005

Served as a writing assistant for Communication Studies 2020, Honors

Fundamentals of Communications; conducted one-on-one sessions with students outside of class; offered written feedback on early drafts of speeches; facilitated class discussion and in-class assignments.

Instructional Aide, ELL Department, Glendale High School, Glendale, AZ, 2003-2004 Tutored students from a variety of cultural backgrounds at all stages of English literacy, including first generation speakers.

\section{SELECTED GRADUATE COURSES}

The University of Louisville

Research in the Composing Process (Joanna Wolfe)

Linguistic Theory and Its Applications (Joanna Wolfe)

Writing, Cognition, and Culture in Curriculum Design (Bruce Horner)

Writing Assessment and Response (Min-Zhan Lu)

Rhetorical and Textual Analysis (Min-Zhan Lu)

Nineteenth-Century American Poetry and Prose (Susan Ryan)

Teaching Composition (Bronwyn Williams)

Creative Writing (Paul Griner)

Introductory Applied Statistics (Namok Choi)

Cultural Rhetoric (Annette Powell)

Linguistic Theory (Karen Hadley)

Composition Theory (Karen Kopelson)

Middle Tennessee State University

Postcolonial Literature and Theory (Newtona Johnson)

Introduction to Literary Theory (Allen Hibbard)

Independent Study on WAC and Writing Fellows (Trixie Smith) 
Writing Center Theory (Trixie Smith)

Teaching Composition (Trixie Smith)

Composition Theory and Professional Development (Allison Smith)

\section{ACADEMIC SERVICE}

Facilitated pedagogy workshop, "Developing Skillful Writing Learners through Course Document Analysis,” Spring 2010.

Member, English Graduate Organization, University of Louisville, 2008-Present

Mentor to first-year doctoral students, 2008

Facilitated pedagogy workshop, "Politics in the Classroom: Teaching the 2008 Election," Fall 2008.

Event Coordinator, Virginia Peck Composition Series. Guest Bronwyn T. Williams. "Life Is Not Like the Movies (Or Is It?): Representations of Literacy in Popular Culture," "Popular Culture and Writing Pedagogy: Engaging What Students Bring to Class," and "Faces, Images, and Words: Identity and Writing in the Composition Classroom." Middle Tennessee State University. February 16, 2007.

Vice President, English Graduate Student Organization, Middle Tennessee State University, 2006-2007.

Member, English Graduate Student Organization, Middle Tennessee State University, 2006-2008 